\title{
Weighted Local Variance-Based Edge Detection and Its Application to Vascular Segmentation in Magnetic Resonance Angiography
}

\author{
Max W. K. Law* and Albert C. S. Chung
}

\begin{abstract}
Accurate detection of vessel boundaries is particularly important for a precise extraction of vasculatures in magnetic resonance angiography (MRA). In this paper, we propose the use of weighted local variance (WLV)-based edge detection scheme for vessel boundary detection in MRA. The proposed method is robust against changes of intensity contrast of edges and capable of giving high detection responses on low contrast edges. These robustness and capabilities are essential for detecting the boundaries of vessels in low contrast regions of images, which can contain intensity inhomogeneity, such as bias field, interferences induced from other tissues, or fluctuation of the speed related vessel intensity. The performance of the WLV-based edge detection scheme is studied and shown to be able to return strong and consistent detection responses on low contrast edges in the experiments. The proposed edge detection scheme can be embedded naturally in the active contour models for vascular segmentation. The WLV-based vascular segmentation method is tested using MRA image volumes. It is experimentally shown that the WLV-based edge detection approach can achieve high-quality segmentation of vasculatures in MRA images.
\end{abstract}

Index Terms-Edge detection, magnetic resonance angiography (MRA), segmentation, vessels, weighted local variance.

\section{INTRODUCTION}

D ETECTING and extracting blood vessels in magnetic resonance angiography (MRA) are critical in reconstructing 3-D vascular models and, thus, essential for clinical assessment of vasculatures. Precise extraction of vessels requires accurate edge detection techniques. To extract blood vessels in the magnetic resonance angiograms, image gradient magnitude is widely used for observing the intensity differences between vessels and background regions. For instance, Malladi et al. [17] proposed to extract vessels by halting contours at positions where the values of $|\nabla G * I|$ are large. Caselles et al. [6] proposed and employed the geodesic active contour to extract blood vessels using a minimal distance curve based on the image gradient magnitude $|\nabla G * I|$. McInerney and Terzopoulos introduced T-Snake [19], which was based on

Manuscript received February 10, 2007; revised June 13, 2007. Asterisk indicates corresponding author.

*M. Law is with the Lo Kwee-Seong Medical Image Analysis Laboratory, Department of Computer Science and Engineering, The Hong Kong University of Science and Technology, Clear Water Bay, Hong Kong. (e-mail: maxlawwk@cse.ust.hk).

A. Chung is with the Lo Kwee-Seong Medical Image Analysis Laboratory, Department of Computer Science and Engineering, The Hong Kong University of Science and Technology, Clear Water Bay, Hong Kong. (e-mail: achung @ cse. ust.hk).

Digital Object Identifier 10.1109/TMI.2007.903231 image gradient magnitude, and used the Laplacian operation to discover boundaries for tissue extraction in medical images.

Along the same research line, not only gradient magnitude, but also gradient direction has been used as feature for the extraction of vessels. Xiang et al. proposed an elastic interaction model [29], [30]. The main concept is to locate vessel boundaries by minimizing an energy term associated with a magnetic field calculated from image gradient. Vasilevskiy and Siddiqi introduced [25] the flux maximizing geometric flows. The vessel boundaries were selected according to the zero-crossing boundaries of the magnetic flux, which was computed from image gradient.

Vessel boundaries can also be detected with the help of structural information in addition to the image gradient. Lorigo et al. presented the CURVES algorithm [14], [15], which extended the gradient magnitude-based geodesic active contour method [6] using the arbitrary codimension framework [1]. The CURVES algorithm contained a heuristic factor. By adjusting this factor, the algorithm can enhance the detection and segmentation of tubular structures. Yan and Kassim also improved the geodesic active contour method [6] by employing capillary effects for detection of thin vessel boundaries [31], [32].

On the other hand, the Hessian matrix-based structural information is also useful in the detection of vessel boundaries. As mentioned in a review by Sato et al. [22], the eigenvalues of the Hessian matrix can provide valuable information about the shape and local structures of a boundary. Frangi et al. introduced the term "vesselness" [9] as a measurement of tubular structures by observing the ratio of eigenvalues of the Hessian matrix. Bullitt et al. [4] presented a work that found the vessel centerlines first and then located the vessel boundaries according to the eigenvalue ratio of the Hessian matrix. Descoteaux et al. [8] employed the vesselness measure [9] to detect the boundaries of tubular structures and incorporated it in [25] to perform segmentation. Westin et al. [27] utilized the Hessian matrix to detect the boundaries of plane or tubular structures and the Hessian matrix-based boundary information was complemented with the codimension two segmentation method [15].

In the aforementioned approaches [4], [8], [9], [22], [27], the structural information of the Hessian matrix is quantified by the relation of eigenvalues along different principle directions of the Hessian matrix. Different from the gradient, which utilizes the first derivatives of an image, the Hessian matrix is based on the second derivatives of images to compute the curvatures of boundaries. The curvature in the normal direction of the boundaries of vessels, which are mainly in tubular shape, 
should be much larger than the curvatures in other principle directions. Compared with the image gradient, which is general and has responses independent of the shape and local structures of boundaries, the Hessian matrix can distinguish between types of boundaries (e.g., tubes, planes, blob surfaces, or noise) so that the Hessian matrix-based techniques can be tailored to the target tubular structures.

For some nontubular vasculatures such as junctions or ending points, they can induce high curvature values along more than one principle directions. This can possibly lead to inaccurate detection of vessel boundaries using the Hessian matrix-based methods. On the other hand, although the image gradient is more general in handling structures with different shapes, due to the presence of intensity inhomogeneity such as bias field, overlapping between vessels and other tissues or the speed related vessel intensity, the intensity difference between vessels and background regions are not consistent but are varying. The boundaries of the low contrast vessels cannot provide large values of the gradient term $|\nabla G * I|$ for the methods based on image gradient to detect those vessel boundaries.

In this paper, we propose a general edge detection approach based on weighted local variances (WLVs), which quantify intensity similarity on both sides of an edge for edge detection. The WLV-based method is robust against changes of intensity contrast between vessels and image background regions, and is able to return strong and consistent edge responses on the boundaries of low contrast vessels. Different from the Hessian matrix-based techniques, which analyze the shape and local structures of boundaries for detection of tubular vascular structures, the proposed WLV-based scheme is a general technique that returns high detection responses on low contrast edges disregarding the shape and local structures of boundaries.

Using the edge detection results of WLV-based method, which include the edge strength and the edge normal direction, blood vessels are extracted by the flux maximizing geometric flows [25]. In the experiments, the edge strength and the edge normal direction computed by the proposed method are studied using two synthetic volumes. The WLV-based vascular segmentation method is validated and compared using a time-of-flight (TOF) MRA and three phase contrast (PC) MRA image volumes. It is experimentally shown that the WLV-based method is capable of giving high and consistent edge strength in low contrast boundaries and the active contour-based segmentation using WLV is able to handle low contrast vessels.

The rest of this paper is organized as follows. The relationship between edge detection and WLV is presented in Section II-A. The analysis of the properties of the WLV is given in Section II-B. In Sections II-C and II-D, the implementation details of the WLV-based edge detection scheme are shown. Section II-E elaborates the procedures for the extraction of vessels based on edge detection results of the WLV-based method. We study the performance of the WLV-based edge detection scheme using two synthetic image volumes in Section III-A. The segmentation results of the proposed method obtained from four clinical cases are presented and compared in Section III-B. In Section IV, the discussion of the proposed method and the future direction of this research are provided. Finally, we conclude this paper in Section V.

\section{Methodology}

\section{A. Edge Detection and Weighted Local Variance (WLV)}

In this section, we introduce the use of WLV for extracting edge information, including edge normal orientation and edge strength. The WLV is a general edge detection technique, which considers the voxel intensity homogeneity within local regions. To extract edge information based on the WLV, we first consider the directional derivative of a Gaussian function. The directional derivative of a Gaussian function $f(\vec{x})$ along a direction $\hat{n}$ at a position $\vec{x}$ in 2-D is given by

$$
\begin{aligned}
f_{\hat{n}}(\vec{x}) & =\frac{\partial f(\vec{x})}{\partial \hat{n}} \\
& =-\frac{\vec{x} \cdot \hat{n}}{2 \pi \sigma^{2} \sqrt{\sigma \sigma_{\perp}}} \exp \left(-\frac{(\vec{x} \cdot \hat{n})^{2}}{2 \sigma^{2}}-\frac{|\vec{x} \times \hat{n}|^{2}}{2 \sigma_{\perp}{ }^{2}}\right)
\end{aligned}
$$

and in 3-D

$$
\begin{aligned}
f_{\hat{n}}(\vec{x}) & =\frac{\partial f(\vec{x})}{\partial \hat{n}} \\
& =-\frac{\vec{x} \cdot \hat{n}}{(2 \pi)^{3 / 2} \sigma^{5 / 2} \sigma_{\perp}} \exp \left(-\frac{(\vec{x} \cdot \hat{n})^{2}}{2 \sigma^{2}}\right. \\
& \left.-\frac{\left(\vec{x} \cdot \overrightarrow{n_{1}}\right)^{2}+\left(\vec{x} \cdot \overrightarrow{n_{2}}\right)^{2}}{2 \sigma_{\perp}{ }^{2}}\right)
\end{aligned}
$$

where $\overrightarrow{n_{1}}$ and $\overrightarrow{n_{2}}$ are the unit vectors, which are perpendicular to each other and orthogonal to $\hat{n}$, mathematically, $\hat{n}=\overrightarrow{n_{1}} \times \overrightarrow{n_{2}}$. These filters are sensitive to an edge having normal direction aligned with $\hat{n}$. The value of $\sigma$ determines the scale of an edge detectable by the filter, while the value of $\sigma_{\perp}$ specifies the size of the filters in directions orthogonal to the derivative direction. In the case that $\sigma \neq \sigma_{\perp}, f(\vec{x})$ is an anisotropic Gaussian function, which is dependent on the orientation $\hat{n}$; when $\sigma=\sigma_{\perp}$, the above equations represent the directional derivatives of an isotropic Gaussian function, which is similar to the filter proposed in [5].

The goal of the WLV is to quantify voxel intensity homogeneity locally based on the directional derivatives of a Gaussian function. To achieve this, we first split $f_{\hat{n}}(\vec{x})$ into two halves

$$
\begin{aligned}
& f_{1, \hat{n}}(\vec{x})=\left\{\begin{array}{ll}
f_{\hat{n}}(\vec{x}), & \text { if } f_{\hat{n}}(\vec{x}) \leq 0 \\
0, & \text { otherwise, }
\end{array}\right. \text { and } \\
& f_{2, \hat{n}}(\vec{x})=\left\{\begin{array}{ll}
f_{\hat{n}}(\vec{x}), & \text { if } f_{\hat{n}}(\vec{x})>0 \\
0, & \text { otherwise }
\end{array} .\right.
\end{aligned}
$$

These two filters are then normalized to be sum-to-one, for $i=$ $\{1,2\}$

$$
g_{i, \hat{n}}(\vec{x})=\frac{f_{i, \hat{n}}(\vec{x})}{\int f_{i, \hat{n}}(\vec{x}) d \vec{x}} .
$$

Using these normalized filters, the value of WLV is calculated. Broadly speaking, variance is a measure to estimate the sparseness of a set of variables. Similarly, based on the normalized filters, around the position $\vec{x}$, the WLV evaluates the intensity homogeneity within two local regions separated by an edge having normal direction aligned with $\hat{n}$. These two local regions are associated with the nonzero entries of $g_{1, \hat{n}}(\vec{x})$ and $g_{2, \hat{n}}(\vec{x})$. Hence, 


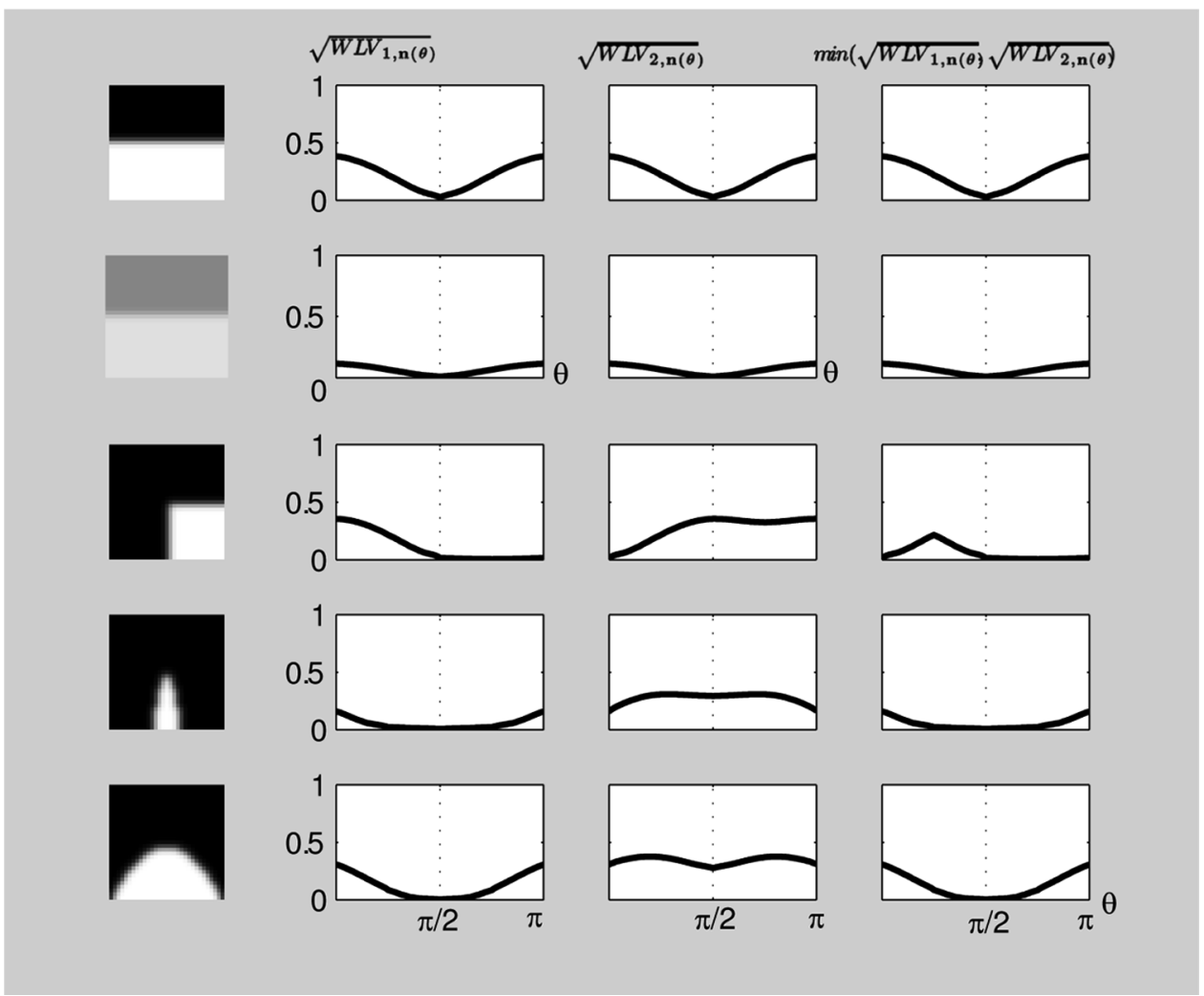

Fig. 1. The plots of the values of $\sqrt{\mathrm{WLV}_{1, \hat{n}(\theta)}}$ (second column), $\sqrt{\mathrm{WLV}_{2, \hat{n}(\theta)}}$ (third column), and min $\left(\sqrt{\mathrm{WLV}_{1, \hat{n}(\theta)}}, \sqrt{\mathrm{WLV}} \mathrm{V}_{2, \hat{n}(\theta)}\right.$ ) (forth column) against different values of $\theta$ obtained from horizontal edges having different levels of intensity contrast, a corner, and two edges with different values of curvature.

the WLVs for both split filters are defined as

$$
\mathrm{WLV}_{i, \hat{n}}=\int g_{i, \hat{n}}(\vec{y})\left(I(\vec{x}+\vec{y})-\mu_{i, \hat{n}}(\vec{x})\right)^{2} d \vec{y}
$$

where $I(\vec{x})$ represents the intensity at $\vec{x}$, and $\mu_{1, \hat{n}}$ and $\mu_{2, \hat{n}}$ are the weighted intensity averages of their corresponding filters, i.e., $\mu_{i, \hat{n}}(\vec{x})=\int g_{i, \hat{n}}(\vec{y}) I(\vec{x}+\vec{y}) d \vec{y}, i=\{1,2\}$.

The WLVs, $\mathrm{WLV}_{1, \hat{n}}$, and $\mathrm{WLV}_{2, \hat{n}}$, are weighted sums of squared intensity differences between the intensities of the neighboring voxels $I(\vec{x}+\vec{y})$ and their corresponding weighted intensity averages, $\mu_{1, \hat{n}}(\vec{x})$ and $\mu_{2, \hat{n}}(\vec{x})$, respectively. As such, the variances aim to evaluate the intensity homogeneity in two local regions separated by an edge. To illustrate the idea, we use five examples consisting of horizontal edges having different levels of intensity contrast, a corner and two edges with different values of curvature. This is shown in Fig. 1. The figure shows the values of $\sqrt{\mathrm{WLV}_{1, \hat{n}(\theta)}}$ and $\sqrt{\mathrm{WLV}_{2, \hat{n}(\theta)}}$ with various orientations, $\hat{n}(\theta)=[x \cos \theta-y \sin \theta, x \sin \theta+y \cos \theta]^{T}$. It demonstrates how WLV varies with the orientation of detection $\hat{n}(\theta)$. As shown in Fig. 1, for all the five examples, the variances vary as $\theta$ changes and attain small values when the corresponding filters, $f_{1, \hat{n}(\theta)}$ or $f_{2, \hat{n}(\theta)}$ along $\theta$, do not cross the edges. It is observed that the value of $\min \left(\sqrt{\mathrm{WLV}_{1, \hat{n}(\theta)}}, \sqrt{\left.\mathrm{WLV}_{2, \hat{n}(\theta)}\right)}\right.$ is small when $\theta$ is approaching the edge normal orientation. A small WLV value implies that the voxel intensities are similar in the two local regions on two different sides of an edge.
Therefore, we define a confidence value for finding an edge having normal orientation $\hat{n}$, as

$$
R_{\hat{n}}(\vec{x})=\frac{\mu_{1, \hat{n}}(\vec{x})-\mu_{2, \hat{n}}(\vec{x})}{\sqrt{\min \left(\mathrm{WLV}_{1, \hat{n}}(\vec{x}), \mathrm{WLV}_{2, \hat{n}}(\vec{x})\right)}+\epsilon}
$$

where the epsilon $\epsilon$ avoids singularity when either or both $\mathrm{WLV}_{1, \hat{n}}(\vec{x})$ and $\mathrm{WLV}_{2, \hat{n}}(\vec{x})$ are zero. The value of this constant is $10^{-3}$ in our implementation. On one hand, the denominator of (5), based on the WLVs for evaluating intensity similarity between both sides of an edge, should be small when an edge is likely to be found. On the other hand, the numerator measuring the intensity change across an edge should be large if an edge is detected. Therefore, a high confidence value implies the presence of an edge having normal orientation $\hat{n}$.

\section{B. Properties}

In the first and second rows of Fig. 1, we show two horizontal edges. The former has intensity values 1 and 0 and the latter with lower contrast has intensity values 0.9 and 0.6 , which give smaller intensity difference across the edge. Comparing the variances obtained from the high contrast edge (the top row of Fig. 1) and the low contrast edge (the second row of Fig. 1), the variances are generally smaller in the case of the lower contrast edge. Since the values of both terms, $\left[\mu_{1, \hat{n}}(\vec{x})-\mu_{2, \hat{n}}(\vec{x})\right]$ and $\min \left(\sqrt{\mathrm{WLV}_{1, \hat{n}(\theta)}}, \sqrt{\left.\mathrm{WLV}_{2, \hat{n}(\theta)}\right)}\right.$, are reduced for low contrast edges, the return value of (5) is not affected significantly by the change of intensity contrast. This concept can be mathematically illustrated by considering two arbitrary image 
patches, $I$ and $J$, which have different levels of intensity contrast and brightness but are related by $I(\vec{x})=c J(\vec{x})+b, c>0$. The terms $c$ and $b$ are constants representing the differences in intensity contrast and brightness, respectively. The numerator of the confidence value in (5) for the image patch, $I$ and $J$, are related as

$$
\begin{aligned}
\mu_{1, \hat{n}}^{I}-\mu_{2, \hat{n}}^{I} & =\int_{\text {since } g_{1} \text { and } g_{2} \text { are summed-to-one }}(c J(\vec{x}+\vec{y})+b)\left(g_{1, \hat{n}}(\vec{y})-g_{2, \hat{n}}(\vec{y})\right) d \vec{y} \\
& =c\left(\mu_{1, \hat{n}}^{J}-\mu_{2, \hat{n}}^{J}\right) .
\end{aligned}
$$

Moreover, for the denominator of the confidence value in (5), we evaluate the WLVs (4) for the image patches, $I$ and $J$, for $i=\{1,2\}$

$$
\begin{aligned}
& \operatorname{WLV}_{i, \hat{n}}^{I}(\vec{x}) \\
& \quad=\int\left\{g_{1, \theta}(\vec{y})\left(c J(\vec{x}+\vec{y})+b-\mu_{i, \hat{n}}^{I}(\vec{x}+\vec{y})\right)^{2} d \vec{y}\right. \\
& =\int\left\{g_{1, \theta}(\vec{y})\left(c J(\vec{x}+\vec{y})+b-c \mu_{i, \hat{n}}^{J}(\vec{x}+\vec{y})-b\right)^{2} d \vec{y}\right. \\
& =c^{2} \operatorname{WLV}_{i, \hat{n}}^{J}(\vec{x}) .
\end{aligned}
$$

Therefore, according to (5), the confidence value of the image patch $I$ is given as

$$
\begin{aligned}
R_{\hat{n}}^{I}(\vec{x}) & =\lim _{\epsilon \rightarrow 0} \frac{c\left[\mu_{1, \hat{n}}^{J}(\vec{x})-\mu_{2, \hat{n}}^{J}(\vec{x})\right]}{c \sqrt{\min \left(\mathrm{WLV}_{1, \hat{n}}^{J}(\vec{x}), \mathrm{WLV}_{2, \hat{n}}^{J}(\vec{x})\right)}+\epsilon} \\
& \approx R_{\hat{n}}^{J}(\vec{x}) .
\end{aligned}
$$

The brightness term $b$ is eliminated and the contrast term $c$ is approximately canceled for the calculation of confidence value defined in (5), except that there is a small constant $\epsilon$.

With regard to the confidence value defined in (5), the numerator (i.e., the intensity difference term) and the denominator (i.e., the WLV terms) measure, respectively, the intensity difference across an edge and intensity similarity between two local regions separated by the edge. Both the intensity difference and the value of WLV are altered according to the contrast term $c$ and this leads to the cancellation of the contrast term in (8). In practice, in the presence of a low contrast edge [i.e., $c$ is small in (6)-(8)], the intensity similarity is high (i.e., the values of WLVs are small), which can compensate the reduced intensity change across low contrast edge. It enables the confidence value in (5) to retain in high level for low contrast edges.

\section{Computing Edge Normal Orientation and Edge Strength}

In the previous section, we introduce a confidence value in (5) based on the WLV for edge detection. In this section, we further elaborate the procedures to obtain edge strength and edge normal orientation using WLV-based confidence values. The WLV-based edge detection method is named WLV-EDGE hereafter to distinguish it from the calculation of WLVs in (4).

Both the edge strength and the edge normal orientation of WLV-EDGE are quantified by calculating the confidence values in a set of discretized orientations. The set of discrete orientations is denoted as a set of unit vectors

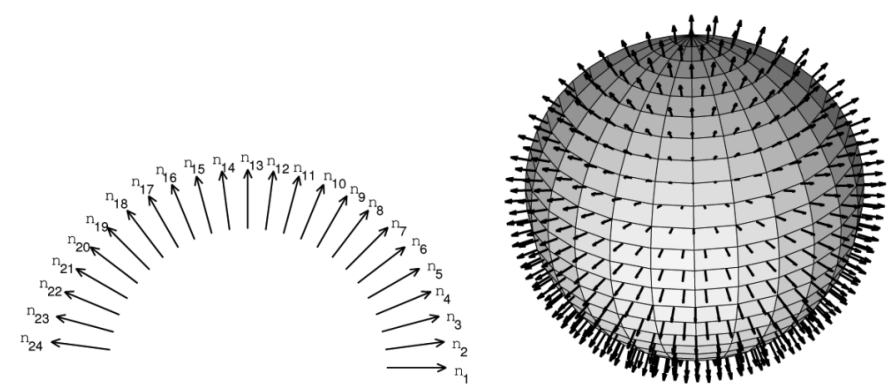

(a)

(b)

Fig. 2. (a) An example of 24 discrete 2-D orientation samples for calculation of confidence values. (b) An example of 281 discrete 3-D orientation samples for calculation of confidence values.

$\hat{n_{k}}, k=\{1,2,3, \ldots, K\} \cdot \hat{n_{k}}$ is the $k$ th discrete orientation sample (see Fig. 2 for the typical sets of 2-D and 3-D orientations). Based on (5), the confidence value obtained along the orientation $\hat{n_{k}}$ is denoted as $R_{\hat{n_{k}}}(\vec{x})$. It is noticed that the orientation samples sweep across a semicircle in 2-D or the surface of a hemisphere in 3-D instead of a complete circle or sphere due to the fact that the confidence value is conjugate, i.e., $R_{\hat{n_{k}}}(\vec{x})=-R_{-\hat{n_{k}}}(\vec{x})$.

Although it is straightforward to estimate the edge normal orientation using the orientation associated with the largest confidence value, it is possible that multiple orientation samples give the maximum confidence value, which can be problematic in determining the edge normal orientation in this situation. Therefore, the edge normal orientation is obtained according to the confidence values computed in different discrete orientations. It is achieved by considering the confidence values as a set of points

$$
\left\{R_{\hat{n_{k}}}(\vec{x}) \hat{n_{k}}\right\}, k=\{1,2,3, \ldots, K\} .
$$

Each voxel has its own point set to represent $K$ confidence values. The point set for each voxel is considered independently. The idea is that an orientation having a large output of $R_{\hat{n_{k}}}(\vec{x})$ is likely to be the edge normal orientation. Thus, the orientation having a large value of $R_{\hat{n_{k}}}(\vec{x}) \hat{n_{k}}$ is represented as a point $R_{\hat{n_{k}}}(\vec{x}) \hat{n_{k}}$ located away from the origin in the Euclidean space. Using this representation, the edge normal direction is estimated by finding an orientation such that the points are mostly spanning away. Such edge normal orientation is found using the first principle direction of the point set. This is accomplished by performing eigendecomposition on a matrix associated with the points

$$
\mathbf{M}(\vec{x})=\mathbf{E}_{k}\left[R_{\hat{n}_{k}}^{2}(\vec{x})\left(\hat{n}_{k} \cdot \hat{n}_{k}^{T}\right)\right]
$$

where $\mathbf{E}(\cdot)$ is the expected value.

Three eigenvectors $\hat{e_{1}}(\vec{x}), \hat{e_{2}}(\vec{x})$, and $\hat{e_{3}}(\vec{x})$ corresponding to three eigenvalues $\lambda_{1}(\vec{x}), \lambda_{2}(\vec{x})$, and $\lambda_{3}(\vec{x})$ are obtained, respectively, where $\left|\lambda_{1}(\vec{x})\right| \geq\left|\lambda_{2}(\vec{x})\right| \geq\left|\lambda_{3}(\vec{x})\right|$. Using the first principle direction, either one of the directions $\hat{e_{1}}(\vec{x})$ or $-\hat{e_{1}}(\vec{x})$ represents the edge normal orientation of the voxel $\vec{x}$. The final decision is based on the sign of the sum of confidence value samples projected along $\hat{e_{1}}(\vec{x})$, which is formulated as

$$
\hat{g}(\vec{x})=\operatorname{sign}\left\{\mathbf{E}_{k}\left[\left[R_{\hat{n}_{k}}(\vec{x}) \hat{n}_{k}\right] \cdot \hat{e_{1}}(\vec{x})\right]\right\} \hat{e_{1}}(\vec{x}) .
$$


Meanwhile, the edge strength is computed as

$$
s(\vec{x})=\sqrt{\lambda_{1}^{2}(\vec{x})+\lambda_{2}^{2}(\vec{x})+\lambda_{3}^{2}(\vec{x})} .
$$

The calculation of the edge strength in the above equation is the $L_{2}$ norm of the matrix $\mathbf{M}(\vec{x})$ in (10). Since the entries of this matrix $\mathbf{M}(\vec{x})$ are based on the confidence values, the edge strength calculated from the above equation inherits the robustness of the confidence values against different edge contrasts [see (8) and the discussion in Section II-B]. Therefore, the estimated edge strength can retain a high value despite low contrast edges.

It should be pointed out that the computations of $\hat{g}(\vec{x})$ and $s(\vec{x})$ are based on the values of WLV, which depend on the shape of the filters $g_{1, \hat{n}}$ and $g_{2, \hat{n}}$, as described in (4). The shape of the filters $g_{1, \hat{n}}$ and $g_{2, \hat{n}}$ are controlled by two parameters $\sigma$ and $\sigma_{\perp}$ [see (1)-(3)]. Here, we here briefly describe the influences of these two parameters and the criteria for setting their values.

For the parameter $\sigma$, it specifies the WLV detection range in a direction perpendicular to an edge that has to be detected. It designates the minimum width of the vessels, which boundaries can be detected by WLV-EDGE. It is because, for those vessels with diameter smaller than or close to $\sigma$, the effective range of the filters $g_{1, \hat{n}}$ and $g_{2, \hat{n}}$ used by $\mathbf{W L V}$ in all orientations is longer than the vessel width. Then, the detection range of either one of the filters encloses the entire width of the target vessel and, consequently, both the voxels of the background region and the target vessel are included inside the detection range. The values of WLV are then boosted significantly even though the filters are properly aligned with the edge orientation. As such, for detecting boundary of vessels with diameter smaller than or close to $\sigma, \mathbf{W L V}$ cannot provide reliable edge information and, thus, WLV-EDGE is adversely affected. It is recommended that the value of $\sigma$ is set with the consideration of the width of the narrowest vessels. For example, the value of $\sigma$ can be set slightly smaller than 1-voxel-length in order to help detect the 1-voxelwidth vessels.

For the parameter $\sigma_{\perp}$, it determines the $\mathbf{W L V}$ detection range along the orientation parallel to the target edge. The value of $\sigma_{\perp}$ is suggested to be similar to the value of $\sigma$, given that the value of $\sigma$ is properly assigned. Otherwise, if the value of $\sigma_{\perp}$ is too large, even though $g_{1, \hat{n}}$ and $g_{2, \hat{n}}$ are aligned with the vessel edge orientation, they can inevitably include background voxels outside the target vessel located in the tangential directions of the edge. Furthermore, it is recommended that $\sigma_{\perp}$ should be at least equal to the longest length of a voxel even if $\sigma$ has a value smaller than the longest length of the voxel, so that the detection range of WLV can include enough voxel samples to provide a reliable measurement for estimating WLV-EDGE. In our experiments presented in Section III, the parameters $\sigma$ and $\sigma_{\perp}$ for the clinical cases were selected according to the criteria described above.

\section{Implementation}

The estimations of edge strength and edge normal direction require probing the confidence value in a set of discrete orientations. It is a computation demanding process as it repeatedly calculates the confidence value for each voxel in different orientations. To speed up the proposed method, in our implementation, the calculation of the confidence value (5) is performed in the frequency domain. First, for the denominator of the (5), the WLVs as stated in (4) are rewritten in the form of convolution, for $i=\{1,2\}$

$$
\begin{aligned}
\mathrm{WLV}_{i, \hat{n}}(\vec{x}) & =\int g_{i, \hat{n}}(\vec{y})\left(I(\vec{x}+\vec{y})-\mu_{i, \hat{n}}(\vec{x})\right)^{2} d \vec{y} \\
& =\int g_{(2-i), \hat{n}}(\vec{y}) I^{2}(\vec{x}-\vec{y}) d \vec{y}-\mu_{i, \hat{n}}^{2}(\vec{x}) \\
& =I^{2}(\vec{x}) * g_{(2-i), \hat{n}}(\vec{x})-\mu_{i, \hat{n}}^{2}(\vec{x})
\end{aligned}
$$

similarly, for the terms $\mu_{i, \hat{n}}$ appeared in the above equation and in the numerator of (5)

$$
\begin{aligned}
\mu_{i, \hat{n}}(\vec{x}) & =\int g_{(2-i), \hat{n}}(\vec{y}) I(\vec{x}-\vec{y}) d \vec{y} \\
& =I(\vec{x}) * g_{(2-i), \hat{n}}(\vec{x}) .
\end{aligned}
$$

Then, the convolution is computed as the multiplication in the frequency domain using the fast Fourier transform algorithm. The running time of calculation of WLVs in a single orientation is reduced significantly from $O\left(N^{2}\right)$ to $O(N \log N)$ for an image with $N$ voxels.

On the other hand, it is always beneficial to have a larger number of orientation samples for WLV-EDGE. However, an excessive use of orientation samples can increase the running time of WLV-EDGE without remarkable enhancement of accuracy. We suggest that the number of orientation samples should be associated with the size of filtering window since a large filtering window extends the effective range of the filtering process, which requires a finer angular resolution.

In 2-D cases, for a semicircle having diameter equal to the dimension of the filtering window, we can have at least one equally spaced orientation sample per unit length for this semicircle. In our implementation, there are 15 voxels in each dimension for the filtering window. The circumference of the corresponding semicircle having diameter 15 is $(15 \pi / 2)$. In order to have at least one equally spaced orientation sample per unit length, we should have $\lceil 15 \pi / 2\rceil$ samples, i.e., 24 samples. An example of the 24 orientation samples in 2-D is shown in Fig. 2(a).

For 3-D cases, the orientation samples are organized in a grid fashion along the longitudinal and the latitudinal directions. There are 24 angularly equally spaced latitudinal levels for the same reason as in 2-D cases. At the latitudinal level of $(\pi / 2)$, there are 24 orientation samples along the longitudinal direction with the aforementioned reason. In each of other latitudinal levels, the number of samples is reduced according to the circumference of semicircles in the corresponding latitudinal levels along the longitudinal direction. As such, there are totally 281 orientation samples, which are roughly equally spaced, as shown in Fig. 2(b).

\section{E. Segmentation Using Active Contour Model}

The edge normal direction and the edge strength computed in (11) and (12) are the edge information of WLV-EDGE. The robustness of WLV-EDGE against changes of intensity contrast 
of edges makes it suitable to detect blood vessel boundaries for active contour-based vascular segmentation.

Due to the nature of vascular images such as the presence of intensity inhomogeneity like bias field, the presence of other tissues or fluctuation of the speed related intensity, it is possible that some vessels have lower intensity contrast than other structures. These low contrast vessels are difficult to detect in active contour models. The difficulty of detection is due to the fact that the boundaries of the low contrast vessels could not exert enough "forces" to compete with other boundaries. It results in contour leakages if the contour is attracted by the edges of other unrelated tissues but not the weak edges of low contrast vessels. On the other hand, it can possibly lead to contours being halted before reaching the vessel boundaries as those boundaries cannot attract the contours to the desired position.

Although the formulation of WLV-EDGE is general and not limited to the detection of vessel boundaries, the edge strength computed from WLV-EDGE is robust to the changes of intensity contrast of edges. The computed edge strength also retains high edge strength even when the edge contrast is low. Therefore, the edge information computed from WLV-EDGE is useful for active contour models to prevent missing of weak edges.

Using the edge information extracted by WLV-EDGE, we apply flux maximizing geometric flows FLUX [25] to incorporate WLV-EDGE for the extraction of blood vessels. There are two reasons that we choose FLUX to complement with WLV-EDGE (it is mentioned as WLV-FLUX hereafter). First, some approaches such as geodesic active contour [6], [12] or geometric snake model [33] do not utilize edge directional information for segmentation. Thus, they are not suitable for the evaluation of the proposed method, which returns both edge strength and edge normal direction. In addition, the proposed technique is independent of tubular structures and does not make use of shape and local structural information. Although there are other methods that employ both edge strength and edge normal direction such as CURVES [14], [15] or capillary active contour [31], [32], these methods aim at detecting tubular structures, which is not the focus of the proposed method. On the other hand, FLUX utilizes both edge strength and edge normal orientation and is not limited to tubular structure. Furthermore, FLUX is the state-of-the-art, which has been used to generate reference segmented vessels for BrainWeb [2]. Therefore, FLUX is employed and incorporated with WLV-EDGE to perform segmentation of blood vessels.

In FLUX, the motion of a closed active contour $\mathcal{C}$ parameterized by $l$ is given by

$$
\mathcal{C}_{t}(l)=\{\nabla \vec{v}(\vec{x}(l))\} \cdot \overrightarrow{\mathcal{N}}(l)
$$

where $\mathcal{N}(l)$ is the normal direction of the contour $\mathcal{C}(l)$ and $\vec{v}(\vec{x}(l))$ is a vector field associated with the gradient. In practice, as described in [25], the divergence of $\vec{v}$ is calculated using a multiscale method and discretized as

$$
\nabla \vec{v}(\vec{x}) \approx \max _{r \in R}\left\{\frac{1}{N} \sum_{n}^{N} \vec{v}\left(\vec{x}+r \cdot \hat{c}_{r, n}\right) \cdot \hat{c}_{r, n}\right\}
$$

where $\hat{c}_{n}$ is the outward normal of the $n$th sample point on the bounding sphere having radius $r$ and centered at $\vec{x}, N$ is the total number of samples taken and $R$ is a set of discrete radii, which specifies the radii of targeted vessels. In our implementation, $N$ was set to 562 (in a sphere fashion), which is the double of $K=281$ (in a hemisphere fashion) for WLV-EDGE in (10) and (11). In [25], Vasilevskiy and Siddiqi have proposed to obtain the vector field $\vec{v}$ as the gradient vector field of an image $I$ smoothed by an isotropic Gaussian filter $G$, i.e.,

$$
\vec{v}(\vec{x})=\nabla(G(\vec{x}) * I(\vec{x})) .
$$

To formulate WLV-FLUX, the edge strength (12) and the edge normal orientation (11) extracted by WLV-EDGE are incorperated in FLUX. As such, the vector field $\vec{v}$ in (17) is substituted using a vector field $\vec{w}(\vec{x})$ based on the edge detection results obtained from WLV-EDGE. The vector field is given by

$$
\vec{w}(\vec{x})=s(\vec{x}) \hat{g}(\vec{x})
$$

where $\vec{s}$ and $\hat{g}$ are the edge strength and the edge normal orientation, respectively, computed from WLV-EDGE in (12) and (11). As such, (16) becomes

$$
\begin{aligned}
\nabla \vec{w}(\vec{x}) & \approx \max _{r \in R}\left\{\frac{1}{N} \sum_{n}^{N} \vec{w}\left(\vec{x}+r \cdot \hat{c}_{r, n}\right) \cdot \hat{c}_{r, n}\right\} \\
& =\max _{r \in R}\left\{\frac{1}{N} \sum_{n}^{N}\left(s\left(\vec{x}+r \cdot \hat{c}_{r, n}\right) \hat{g}\left(\vec{x}+r \cdot \hat{c}_{r, n}\right) \cdot \hat{c}_{r, n}\right)\right\} .
\end{aligned}
$$

The zero level of a level set function [21] is utilized to represent the moving contour $\mathcal{C}$. To perform segmentation, the motion of the level set $\phi$ function is governed by

$$
\frac{\partial \phi}{\partial t}=F|\nabla \phi|+\beta \nabla\left(\frac{\nabla \phi}{|\nabla \phi|}\right)
$$

where $F$ is a function that determines the speed of the contour evolving in the normal direction, the second term $\nabla((\nabla \phi) /(|\nabla \phi|))$ is the mean curvature of the level set function, and $\beta$ is a regularization term to specify resultant contour smoothness. The values of $F$ are $\nabla \vec{v}(\vec{x})$ (16) for FLUX and $\nabla \vec{w}(\vec{x})(19)$ for WLV-FLUX.

The discretization scheme and related parameters of (20) follow the sparse field level set method presented in [28]. The implementation is based on the Insight Segmentation and Registration ToolKit (ITK) [11]. For those entries of $\vec{v}$ and $\vec{w}$ in (16) and (19) having noninteger coordinates, the values are linearly interpolated.

\section{EXPERIMENTS}

To validate the performance of WLV-EDGE, we have carried out two sets of experiments on both synthetic and clinical image volumes. The first set of experiments employs three synthetic image volumes with synthetic tubes and tori of various sizes to analyze the estimation accuracy of the edge strength and the edge normal direction under the effect of intensity variation (see Section III-A). The estimation accuracy of WLV-EDGE 


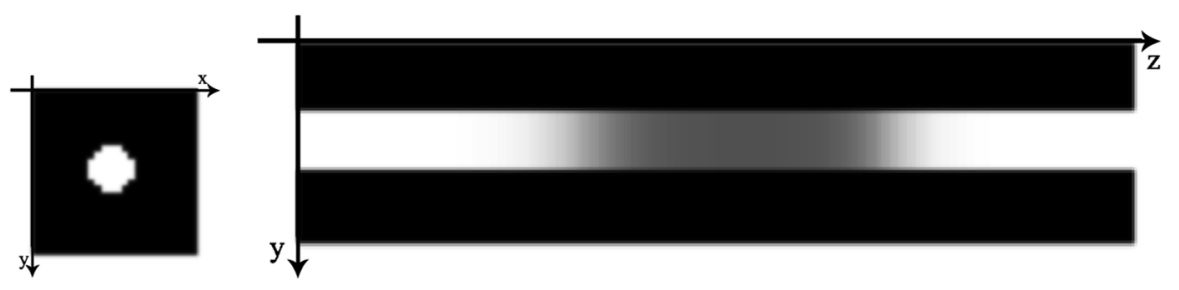

(a)

(b)
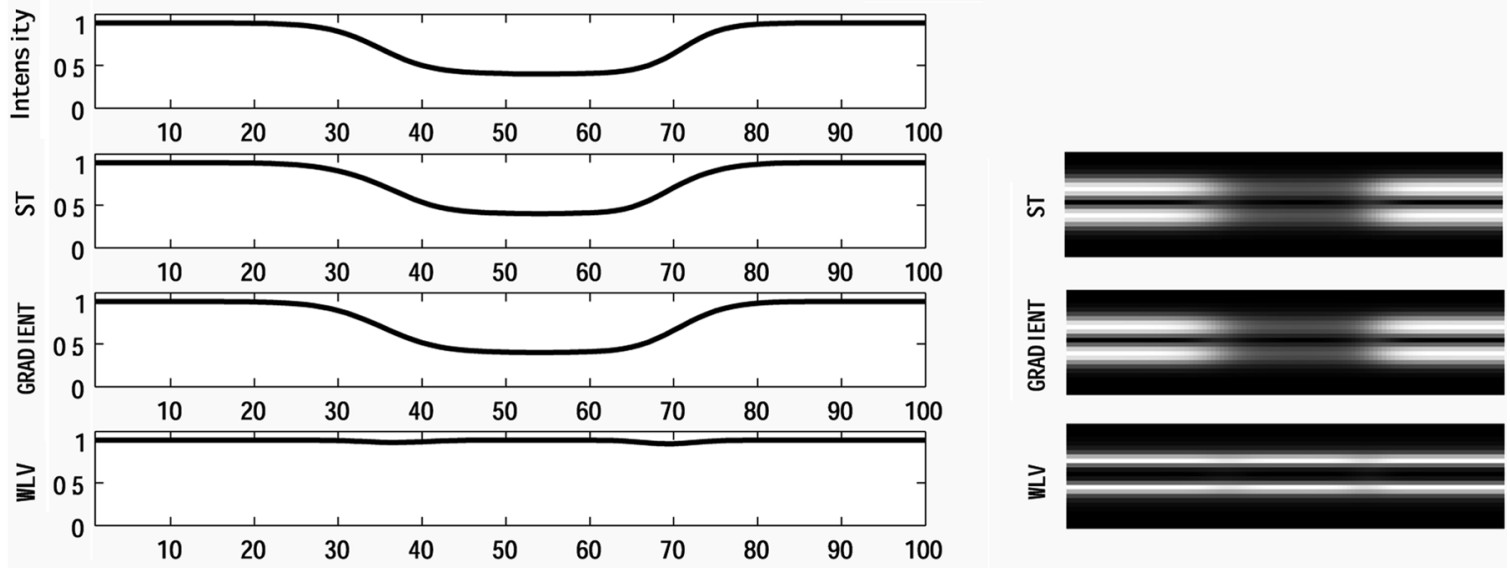

(c)

(d)

Fig. 3. Single synthetic tube. (a) The 50th slice of the image volume, $z=50$. (b) The 12 th slice of the image volume, $x=12$. (c) From top to bottom: The intensity profile of the tube along the $z$-direction with $x=12$ and $y=10$; The square root of the trace of the structure tensor computed by ST; The GRADIENT magnitude; The edge strength computed by WLV. (d) Along the $z$-direction with $x=12$, it shows the slices of edge strengths detected by (from top to bottom) ST; GRADIENT; WLV.

is compared with two edge detection approaches. In the second set of experiments, using four magnetic resonance angiographic image volumes, the performance of WLV-FLUX is compared with FLUX (see Section III-B).

\section{A. Synthetic Image Volumes}

In this section, we present the results obtained from the first experiment, in which a synthetic and numerical image volume of size $24 \times 24 \times 100$ voxels was created. The image volume contains a synthetic and 3-D tube with the diameter of 7 voxels. Fig. 3(a) shows one of the image slices ( $x$ and $y$ axes are also shown). In the image volume, the intensity values inside the tube are consistent in the $x-y$ plane. Although there is no intensity variation in the $x$ - and $y$ - directions, the $x$ and $y$ dimensions are necessary for creating the surface curvature of a 3-D tube. Along the $z$-direction with $x=12$, Fig. 3(b) shows the cross section of the image volume and that the synthetic tube runs from the first slice (left) to the last slice (right). The intensity value is 0 in the background regions and varies along the tube (i.e., along the $z$ axis). The intensity profile along the tube surface ( $x=12$ and $y=10)$ is plotted in the top row of Fig. 3(c). This experiment aims to demonstrate the relationship between the estimated edge strength and the intensity contrast on the tube surface, and the robustness of the proposed method against intensity change along a tube.

For our approach, the edge strength of WLV-EDGE is estimated using (12). The estimated edge strength is then compared with the estimated edge strengths computed from two other edge detection approaches, structure tensor (ST) [3] and smoothed image gradient (GRADIENT). For ST,

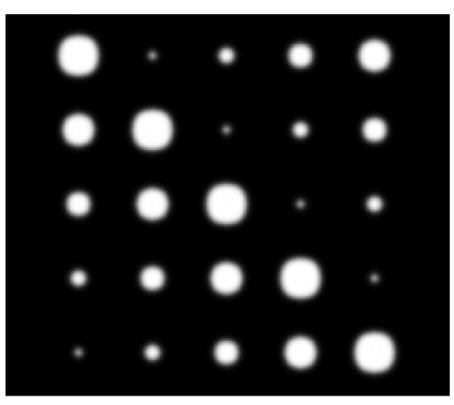

(a)

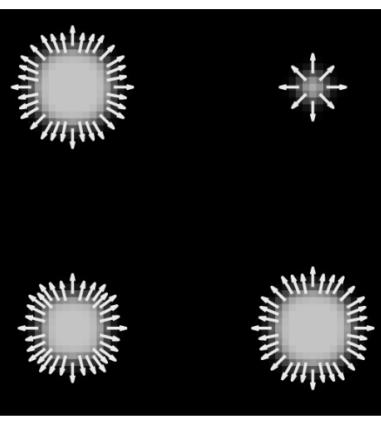

(b)

Fig. 4. Multiple synthetic tubes. (a) The 50th slice of the image volume $(181 \times 217 \times 181$ voxels $)$ consists of 25 synthetic tubes with radii equal to 2 , $3,4,5$, and 6. (b) An example of the ground truth normal orientations on the tube surfaces.

the edge strength is defined as the square root of the trace of the structure tensor. The edge strength of GRADIENT is the magnitude of the filtering responses of applying the directional derivatives of a Gaussian function on an image volume, i.e., $\sqrt{\left(I * G_{x}\right)^{2}+\left(I * G_{y}\right)^{2}+\left(I * G_{z}\right)^{2}}$. In the experiments, for ST, a numerical scheme of central finite difference was utilized to compute the image derivatives for the entries of the structure tensor. The image volume was preprocessed with a Gaussian smoothing filter prior to the calculation of the image derivatives. The value of $\sigma$ used in WLV-EDGE and the scale parameter of the Gaussian filter used in ST and GRADIENT were fixed to 2 . The window size for the filtering processes of ST, GRADIENT and WLV-EDGE was $15 \times 15 \times 15$ voxels. Along the $z$-direction with $x=12$ and $y=10$, the estimated 


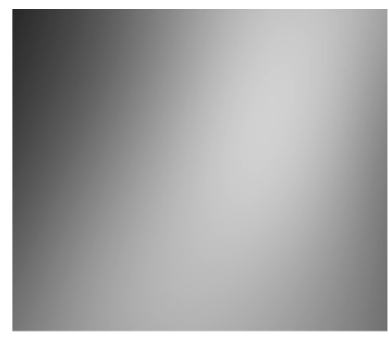

(a)

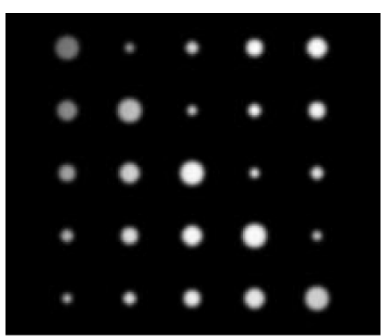

(b)

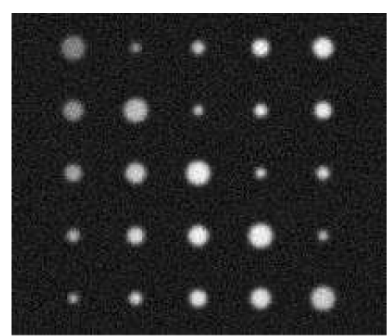

(c)

Fig. 5. (a) The 50th slice of the bias field A obtained from the Brainweb [7]. (b) The 50th slice of the image volume after applying the bias field model A without noise. (c) The 50th slice of the image volume after applying the bias field model A and the additive Gaussian noise with the scale parameter equal to 0.05 .

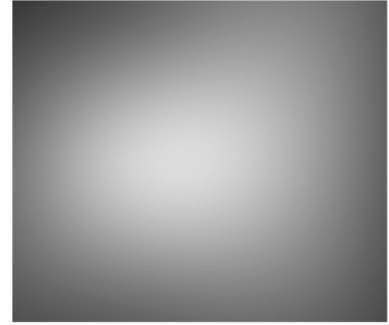

(a)

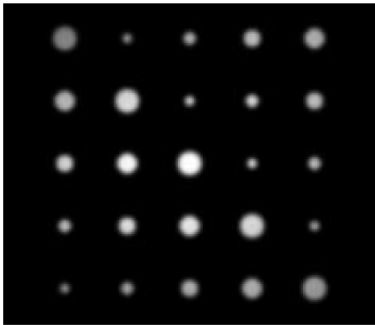

(b)

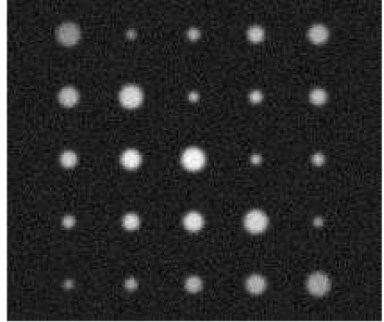

(c)

Fig. 6. (a) The 50th slice of the bias field B obtained from the Brainweb [7]. (b) The 50th slice of the image volume after applying the bias field model B without noise. (c) The 50th slice of the image volume after applying the bias field model B and the additive Gaussian noise with the scale parameter equal to 0.05 .

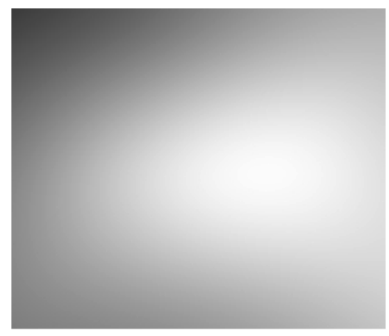

(a)

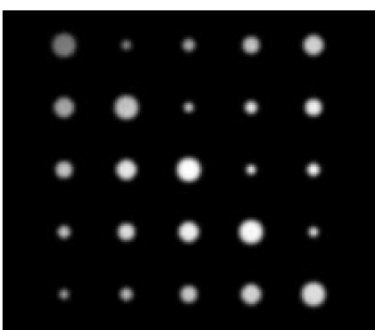

(b)

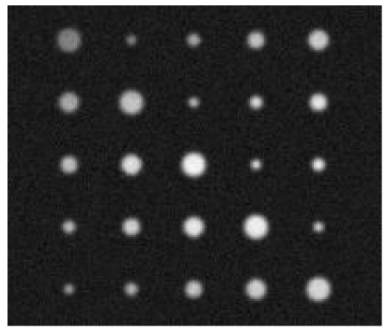

(c)

Fig. 7. (a) The 50th slice of the bias field $\mathrm{C}$ obtained from the Brainweb [7]. (b) The 50th slice of the image volume after applying the bias field model $\mathrm{C}$ without noise. (c) The 50th slice of the image volume after applying the bias field model $\mathrm{C}$ and the additive Gaussian noise with the scale parameter equal to 0.05 .

edge strengths for all the approaches are plotted along with the intensity profile, as shown in Fig. 3(c). The corresponding slices of the edge strengths estimated by the three methods along the $z$-direction with $x=12$ are shown in Fig. 3(d). For comparison, the values of the plots and the slices of the edge strengths were normalized to have maximum value equal to 1 . In the second and third rows in Fig. 3(c), it is observed that the edge strength profiles of ST and GRADIENT fluctuate according to the intensity change along the tube. Conversely, as plotted in the last row of Fig. 3(c), although there are two slight drops of edge strength computed from WLV-EDGE at the positions of 40 and 70, WLV-EDGE's edge strength profile is relatively flat and consistent along the tube, as compared with other two methods. These two slight drops of edge strength were caused by the fact that the estimated confidence values at these two positions were reduced slightly. It is because, in the denominator of (5), the values of WLVs are increased at the positions where the intensity values are varying significantly.

The experimental results show that, as compared with the edge strength computed from ST and GRADIENT, the esti- mated edge strength of WLV-EDGE is relatively consistent given the intensity inhomogeneity along the tube. It is also observed that the edge strengths computed from ST and GRADIENT follow the trend of the intensity profile along the tube. On the contrary, WLV-EDGE obtains a fairly flat edge strength profile. It is because the intensity differences between the tube and background are normalized by WLV in the calculation of confidence value using (5), as illustrated in (8) and discussed in Section II-B.

In the second experiment, two synthetic and numerical image volumes were created and utilized. Both volumes have the size of $181 \times 217 \times 181$ voxels and are shown in Fig. 4(a) and Fig. 8 . This experiment aims to further study the variation of estimated edge strength and the discrepancy of edge normal orientation estimated by ST, GRADIENT and WLV-EDGE under the effect of intensity inhomogeneity. Different from the first experiment, the intensity inhomogeneity of this experiment was specified according to different bias field models appeared in MR images [23]. Bias fields are inherent to MR imaging and they can cause smooth and slow intensity changes within different 


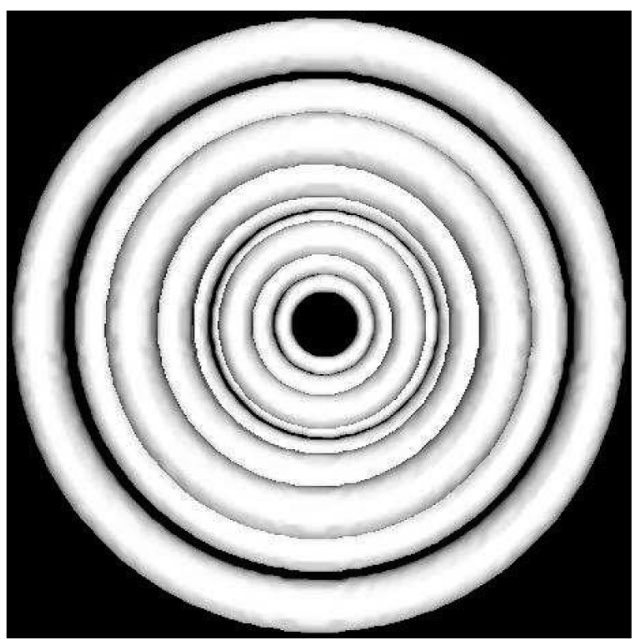

(a)

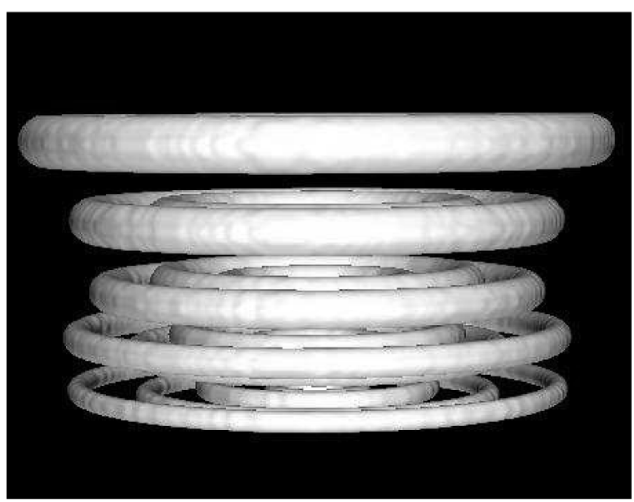

(c)

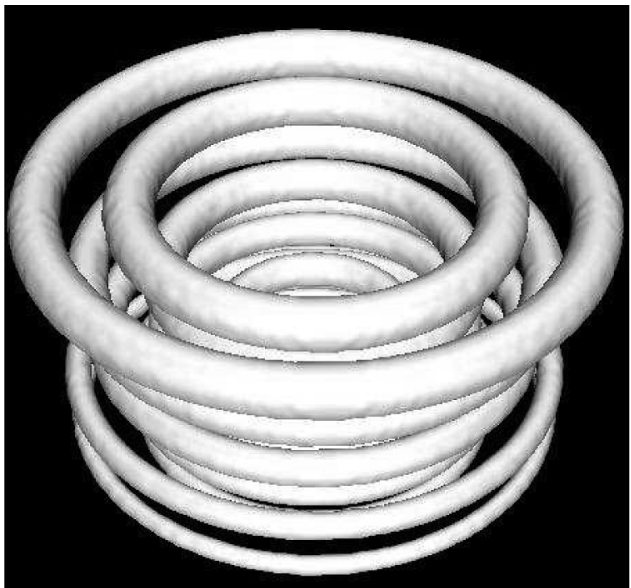

(b)

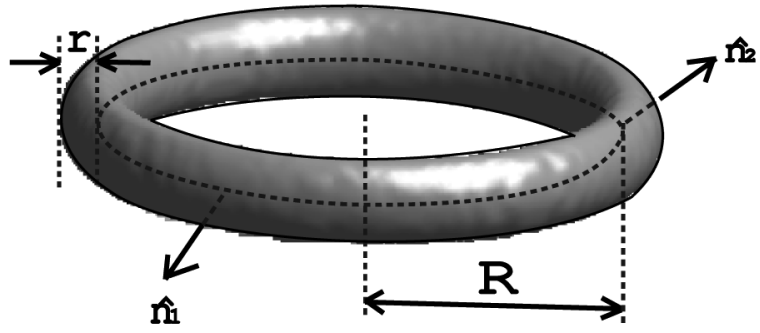

(d)

Fig. 8. The third synthetic and numerical volume consists of 18 nonoverlapping tori. The size of each torus is defined by two parameters $r$ and $R$, as shown in

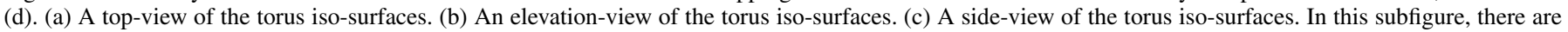

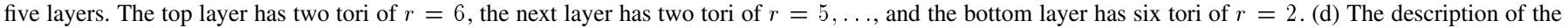

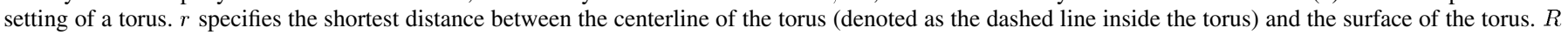
determines the radius of the torus centerline. $\hat{n}_{1}$ and $\hat{n}_{2}$ are two examples of the ground truth normal orientations of the torus surface. They are pointing outward

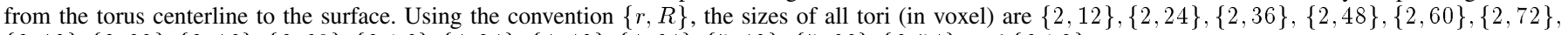
$\{3,18\},\{3,32\},\{3,46\},\{3,60\},\{3,76\},\{4,24\},\{4,48\},\{4,64\},\{5,48\},\{5,66\},\{6,54\}$, and $\{6,72\}$.

regions of an image. This intensity change induced by the bias field is multiplicative and causes variation of intensity contrast between vessels and background.

One of these two synthetic and numerical volumes consists of straight tubes with different radii and the other volume contains tori with various sizes. In the volume of straight tubes, as shown in Fig. 4(a), there are a total of 25 circular tubes having radii equal to $2,3,4,5$, and 6 voxels. This image was formed by first assigning intensity value 1 for the voxels inside the tubes and 0 for the background regions, and then smoothing using a Gaussian filter with a scale parameter equal to 1 . The smoothing aims at creating smooth intensity transitions from the interior of the tubes to the image background. For the volume of tori, as shown in Fig. 8, there are totally 18 nonoverlapping tori. The radius [ $R$ in Fig. 8(d)] of the torus centerline ranges from 12 to 72 voxels, and the shortest distance $[r$ in Fig. 8(d)] between the torus surface and the torus centerline ranges from 2 to 6 voxels. Similar to the straight tube volume, this image was formed by first assigning intensity value 1 for the voxels inside the tori and 0 for the background regions, and then smoothing using a
Gaussian filter with a scale parameter equal to 1 . These tubes and tori, with different sizes, are located in different positions of the images in order to examine how the bias fields affect the performance of different methods.

In this experiment, the bias fields were applied according to the bias field model for MR imaging described in the work [23]. In this model, the resultant signal $s(\mathbf{x})$, the original signal $o(\mathbf{x})$, the noise induced by the imaging device $n(\mathbf{x})$, and the bias field $b(\mathbf{x})$ at position $\mathbf{x}$ are related as

$$
s(\mathbf{x})=o(\mathbf{x}) b(\mathbf{x})+n(\mathbf{x})
$$

The noise generated by the imaging devices, $n(\mathbf{x})$, was simulated by an additive Gaussian noise with $\sigma=0.05$ in order to mimic the noise level observed in the clinical images. The standard deviation of the observed background region intensity in the clinical images was approximately $5 \%$ of the voxel intensity of the brightest vessel. The bias field term $b(\mathbf{x})$ was obtained from the BrainWeb [7], and had three different models, namely, FIELDA, FIELDB, and FIELDC. All of the 
bias fields had 20\% INU. Slices of these bias fields are shown in the Fig. 5(a), Fig. 6(a), and Fig. 7(a), respectively. The slices of the synthetic volume after applying the bias fields are shown in Fig. 5(b) and (c), Fig. 6 (b) and (c), and Fig. 7(b) and (c).

In this experiment, WLV-EDGE is compared with ST and GRADIENT using the same set of parameters as in the previous experiment. The comparison of performance is based on two criteria: (a) the edge strength variation and (b) the edge normal orientation estimation accuracy. For the first criterion, the edge strength variation is quantified by evaluating the standard deviation of edge strengths estimated by different methods on the tube surfaces. A large value of standard deviation of a method implies that the method performance can be easily affected by the bias fields. In contrast, a method is robust to the change of intensity contrast if a small value of standard deviation is observed. Considering different approaches returning edge strengths in different magnitude scales, for a fair comparison, the edge strengths computed from each method were normalized to have unit mean values. Similar to the previous experiment, the edge strengths of ST, GRADIENT and WLVEDGE were calculated using the square root of the trace of the structure tensor, $\sqrt{\left(I * G_{x}\right)^{2}+\left(I * G_{y}\right)^{2}+\left(I * G_{z}\right)^{2}}$ and (12), respectively.

For the second criterion, the measurement of estimation accuracy is based on angular discrepancy between the estimated edge normal orientation and the ground truth normal orientation of the tube or torus surfaces. The ground truth orientation of the tube surfaces is defined as the direction pointing outward from the tube centerline to the tube surface or the torus centerline to the torus surface. Fig. 4(b) and Fig. 8(d) show the ground truth normal orientations of the tubes and tori, respectively. The angular discrepancy is measured in radian as

$$
\arccos (|(\hat{n} \cdot \hat{g})|)
$$

where $\hat{n}$ and $\hat{g}$ are the unit vectors of the estimated edge normal direction and the ground truth normal orientation, respectively. In ST, the surface normal direction is obtained based on the first principle direction of the structure tensor. The surface normal direction of GRADIENT is computed using

$$
\begin{aligned}
& \frac{1}{\sqrt{\left(I * G_{x}\right)^{2}+\left(I * G_{y}\right)^{2}+\left(I * G_{z}\right)^{2}}} \\
& \times\left(I * G_{x}, I * G_{y}, I * G_{z}\right)^{T} .
\end{aligned}
$$

For WLV-EDGE, the edge normal direction is computed according to (11).

In Tables I and II, we list the standard deviations of the estimated edge strength computed by ST, GRADIENT, and WLVEDGE after the bias fields, FIELDA, FIELDB, and FIELDC, have been applied. Each subcolumn in Table I represents the results of ST, GRADIENT, and WLV-EDGE with the tubes having the same radius but at different positions. Also, each subcolumn in Table II represents the results of ST, GRADIENT, and WLV-EDGE with the tori having the same $r$ but with different $R$ and at different positions. From the tables, it is observed that WLV-EDGE consistently gives the smallest standard deviation. This shows that, as compared with ST and GRADIENT,
TABLE I

Standard DeViations of EdGe STRENGTHS COMPUted by DifFERENT METHOdS AFTER APPLYING DifFERENT BIAS FIELd MODELS FIELDA, FIELDB, AND FIELDC ON THE Volume SHOWN IN Fig. 4. THE EDGE STRENGTHS COMPUTEd From EACH METHOD, ST, GRADIENT, AND WLV-EDGE, ARE Normalized to HaVe Unit MEAN VALUES

\begin{tabular}{|c|c|c|c|}
\hline Bias field models & FIELDA & FIELDB & FIELDC \\
\hline \multicolumn{4}{|c|}{ Tube radius $=2$ voxels } \\
\hline ST & 0.2317 & 0.2253 & 0.2400 \\
GRADIENT & 0.2326 & 0.2206 & 0.2316 \\
WLV-EDGE & 0.1701 & 0.1589 & 0.1581 \\
\hline \multicolumn{4}{|c|}{ Tube radius $=3$ voxels } \\
\hline ST & 0.1751 & 0.1692 & 0.2001 \\
GRADIENT & 0.1792 & 0.1752 & 0.1995 \\
WLV-EDGE & 0.1344 & 0.1353 & 0.1350 \\
\hline \multicolumn{4}{|c|}{ Tube radius $=4$ voxels } \\
\hline ST & 0.1224 & 0.1153 & 0.1428 \\
GRADIENT & 0.1354 & 0.1043 & 0.1474 \\
WLV-EDGE & 0.0874 & 0.0608 & 0.0997 \\
\hline \multicolumn{4}{|c|}{ Tube radius $=5$ voxels } \\
\hline ST & 0.1599 & 0.1574 & 0.1685 \\
GRADIENT & 0.1664 & 0.1501 & 0.1731 \\
WLV-EDGE & 0.1010 & 0.0653 & 0.0831 \\
\hline \multicolumn{4}{|c|}{ Tube radius $=6$ voxels } \\
\hline ST & 0.1720 & 0.1602 & 0.1984 \\
GRADIENT & 0.1764 & 0.1501 & 0.2045 \\
WLV-EDGE & 0.1010 & 0.0653 & 0.0857 \\
\hline
\end{tabular}

TABLE II

Standard DeVIations of Edge StRENGTHS COMPUTEd By DifFERENT Methods After APPlying DifFerent BiAs Field Models FIELDA, FIELDB, AND FIELDC ON THE Volume SHOWN IN Fig. 8. THE EdGE STRENGTHS COMPUTED From EACH METHOD, ST, GRADIENT, AND WLV-EDGE, ARE NoRMALIZED to HaVe Unit MEAN VALUES

\begin{tabular}{|c|c|c|c|}
\hline Bias field models & FIELDA & FIELDB & FIELDC \\
\hline Tori, $r=2, R=12,24,36,48,60,72$ voxels \\
\hline ST & 0.1611 & 0.0795 & 0.1371 \\
GRADIENT & 0.1460 & 0.0855 & 0.1100 \\
WLV-EDGE & 0.1345 & 0.0490 & 0.0866 \\
\hline \multicolumn{4}{|c|}{ Tori, $r=3, R=18,32,46,60,76$ voxels } \\
\hline ST & 0.0837 & 0.0844 & 0.1032 \\
GRADIENT & 0.0929 & 0.0864 & 0.1100 \\
WLV-EDGE & 0.0633 & 0.0630 & 0.0527 \\
\hline \multicolumn{4}{|c|}{ Tori, $r=4, R=24,48,64$ voxels } \\
\hline ST & 0.1005 & 0.0764 & 0.0843 \\
GRADIENT & 0.0894 & 0.0808 & 0.0928 \\
WLV-EDGE & 0.0648 & 0.0645 & 0.0734 \\
\hline \multicolumn{4}{|c|}{ Tori, $r=5, R=48,66$ voxels } \\
\hline ST & 0.0941 & 0.1321 & 0.1285 \\
GRADIENT & 0.1045 & 0.1057 & 0.1136 \\
WLV-EDGE & 0.0998 & 0.1001 & 0.1078 \\
\hline \multicolumn{4}{|c|}{ Tori, $r=6, R=54,72$ voxels } \\
\hline ST & 0.1304 & 0.1128 & 0.1066 \\
GRADIENT & 0.1275 & 0.1003 & 0.0981 \\
WLV-EDGE & 0.0800 & 0.0732 & 0.0816 \\
\hline
\end{tabular}

WLV-EDGE is more robust for estimating edge strength in the presence of intensity variation.

For the edge strength analysis, it is noticed that there is a relationship between GRADIENT and WLV-EDGE when $\sigma=\sigma_{\perp}$ (see Appendix A). This relationship also exists in the above synthetic experiments, in which $\sigma=\sigma_{\perp}=2$. As shown in Appendix A, except the multiplicative constant $Z^{-1}$ in (26), the main difference between WLV-EDGE and GRADIENT is the denominator of (26), $\min \left(\sqrt{\mathrm{WLV}_{1, \hat{n}(\theta)}}, \sqrt{\mathrm{WLV}_{2, \hat{n}(\theta)}}\right)$. Therefore, for the edge strength computed by WLV-EDGE, the difference of intensity contrast between tubes and background regions is normalized by this denominator. Thus, different from GRADIENT, the edge strength computed by WLV-EDGE is 
TABLE III

Angular Discrepancies (IN Radian) Between Ground Truth Normal Orientation and the Edge Normal Orientation ESTIMATED by ST, GRADIENT AND WLV-EDGE on the SURFACES OF THE SYNTHETIC TUBES, AS SHOWN IN Fig. 4

\begin{tabular}{|c|c|c|c|c|}
\hline Bias field models & No bias field & FIELDA & FIELDB & FIELDC \\
\hline \multicolumn{7}{|c|}{ Tube radius = 2 voxels } \\
\hline ST & $0.0186(0.0099)$ & $0.0221(0.0180)$ & $0.0139(0.0085)$ & $0.0130(0.0084)$ \\
GRADIENT & $0.0205(0.0093)$ & $0.0225(0.0092)$ & $0.0138(0.0071)$ & $0.0129(0.0018)$ \\
WLV-EDGE & $0.0162(0.0073)$ & $0.0201(0.0077)$ & $0.0099(0.0052)$ & $0.0110(0.0020)$ \\
\hline \multicolumn{7}{|c|}{ Tube radius = 3 voxels } \\
\hline ST & $0.0155(0.0103)$ & $0.0222(0.0132)$ & $0.0107(0.0053)$ & $0.0112(0.0063)$ \\
GRADIENT & $0.0169(0.0075)$ & $0.0216(0.0070)$ & $0.0124(0.0058)$ & $0.0114(0.0060)$ \\
WLV-EDGE & $0.0130(0.0058)$ & $0.0168(0.0058)$ & $0.0091(0.0042)$ & $0.0104(0.0073)$ \\
\hline \multicolumn{7}{|c|}{ Tube radius = 4 voxels } \\
\hline ST & $0.0142(0.0088)$ & $0.0198(0.0093)$ & $0.0156(0.0091)$ & $0.0125(0.0078)$ \\
GRADIENT & $0.0169(0.0090)$ & $0.0202(0.0068)$ & $0.0161(0.0062)$ & $0.0128(0.0062)$ \\
WLV-EDGE & $0.0123(0.0055)$ & $0.0148(0.0051)$ & $0.0104(0.0038)$ & $0.0125(0.0083)$ \\
\hline \multicolumn{7}{|c|}{ Tube radius = 5 voxels } \\
\hline ST & $0.0246(0.0114)$ & $0.0285(0.0123)$ & $0.0296(0.0145)$ & $0.0252(0.0131)$ \\
GRADIENT & $0.0268(0.0111)$ & $0.0282(0.0107)$ & $0.0310(0.0148)$ & $0.0260(0.0112)$ \\
WLV-EDGE & $0.0230(0.0099)$ & $0.0240(0.0124)$ & $0.0296(0.0155)$ & $0.0242(0.0117)$ \\
\hline \multicolumn{7}{|c|}{ Tube radius = 6 voxels } \\
\hline ST & $0.0240(0.0106)$ & $0.0243(0.0166)$ & $0.0240(0.0124)$ & $0.0232(0.0126)$ \\
GRADIENT & $0.0248(0.0103)$ & $0.0266(0.0103)$ & $0.0246(0.0120)$ & $0.0222(0.0109)$ \\
WLV-EDGE & $0.0205(0.0089)$ & $0.0216(0.0090)$ & $0.0224(0.0114)$ & $0.0200(0.0089)$ \\
\hline
\end{tabular}

TABLE IV

Angular Discrepancies (IN RAdian) Between Ground Truth Normal Orientation and the Edge Normal Orientation ESTIMATED By ST, GRADIENT, AND WLV-EDGE ON THE SURfACES OF THE SYNTHETIC TUBES, AS SHOWN IN Fig. 8

\begin{tabular}{|c|c|c|c|c|}
\hline Bias field models & No bias field & FIELDA & FIELDB & FIELDC \\
\hline \multicolumn{7}{|c|}{ Tori, $r=2, R=12,24,36,48,60,72$ voxels } \\
\hline ST & $0.0340(0.0184)$ & $0.0342(0.0187)$ & $0.0340(0.0186)$ & $0.0338(0.0183)$ \\
GRADIENT & $0.0339(0.0182)$ & $0.0337(0.0179)$ & $0.0326(0.0180)$ & $0.0332(0.0178)$ \\
WLV-EDGE & $0.0311(0.0179)$ & $0.0320(0.0176)$ & $0.0319(0.0178)$ & $0.0311(0.0176)$ \\
\hline \multicolumn{6}{|c|}{ Tori, $r=3, R=18,32,46,60,76$ voxels } \\
\hline ST & $0.0232(0.0142)$ & $0.0240(0.0146)$ & $0.0241(0.0147)$ & $0.0242(0.0145)$ \\
GRADIENT & $0.0242(0.0139)$ & $0.0265(0.0136)$ & $0.0263(0.0137)$ & $0.0265(0.0139)$ \\
WLV-EDGE & $0.0236(0.0130)$ & $0.0250(0.0139)$ & $0.0243(0.0130)$ & $0.0249(0.0136)$ \\
\hline \multicolumn{7}{|c|}{ Tori, $r=4, R=24,48,64$ voxels } \\
\hline ST & $0.0241(0.0126)$ & $0.0250(0.0134)$ & $0.0250(0.0130)$ & $0.0249(0.0126)$ \\
GRADIENT & $0.0247(0.0126)$ & $0.0257(0.0134)$ & $0.0250(0.0133)$ & $0.0258(0.0140)$ \\
WLV-EDGE & $0.0237(0.0125)$ & $0.0239(0.0126)$ & $0.0234(0.0122)$ & $0.0234(0.0126)$ \\
\hline \multicolumn{7}{|c|}{ Tori, $r=5, R=48,66$ voxels } \\
\hline ST & $0.0211(0.0128)$ & $0.0215(0.0124)$ & $0.0219(0.0135)$ & $0.0220(0.0136)$ \\
GRADIENT & $0.0225(0.0118)$ & $0.0227(0.0118)$ & $0.0229(0.0118)$ & $0.0229(0.0117)$ \\
WLV-EDGE & $0.0208(0.0101)$ & $0.0207(0.0112)$ & $0.0210(0.0110)$ & $0.0209(0.0111)$ \\
\hline \multicolumn{7}{|c|}{ Tori, $r=6, R=54,72$ voxels } \\
\hline ST & $0.0227(0.0129)$ & $0.0225(0.0134)$ & $0.0225(0.0134)$ & $0.0227(0.0135)$ \\
GRADIENT & $0.0231(0.0130)$ & $0.0228(0.0128)$ & $0.0229(0.0129)$ & $0.0228(0.0128)$ \\
WLV-EDGE & $0.0220(0.0120)$ & $0.0221(0.0125)$ & $0.0220(0.0125)$ & $0.0221(0.0126)$ \\
\hline
\end{tabular}

robust to intensity inhomogeneity induced by the multiplicative effect of bias fields. In contrast, ST and GRADIENT are based on differential operators and can give relatively large variation of edge strength under the effect of bias fields. For the analysis of edge normal orientation estimation, as listed in the WLV-EDGE rows of Tables III and IV for tubes having different radii, tori having different values of $r$ and $R$, and under the effects of different bias fields, WLV-EDGE produces slightly smaller discrepancies than ST and GRADIENT. As a conclusion, WLV-EDGE offers superior robustness of edge strength estimation against intensity inhomogeneity without sacrificing the accuracy of edge normal orientation estimation.

\section{B. Clinical Image Volumes for Segmentation Experiments}

We present and compare the segmentation results obtained from WLV-FLUX and FLUX using four clinical image volumes, a TOF-MRA image volume (Fig. 9) having the size of $188 \times 168 \times 39$ voxels; and three PC-MRA speed image volumes (Fig. 12, Fig. 15, and Fig. 18) having the sizes of $130 \times 286 \times 52$ voxels, $67 \times 257 \times 35$ voxels, and $104 \times 252 \times 64$ voxels, respectively. The third image volume has voxel size $0.4 \mathrm{~mm} \times 0.4 \mathrm{~mm} \times 0.8 \mathrm{~mm}$, and for the rest of image volumes, they have voxel size $0.4 \mathrm{~mm} \times 0.4 \mathrm{~mm} \times 1.0$ $\mathrm{mm}$. All the data sets were acquired using a Philips 3T ACS Gyroscan MR scanner at the University Hospital of Zurich, Switzerland. All image volumes are axial brain scans. The standard TOF-MRA and PC-MRA imaging protocols were used without contrast agents. Details are as follows. For the first volume, $\mathrm{TE} / \mathrm{TR}=3.4 / 23 \mathrm{~ms}$ and flip angle $=20^{\circ}$. For the second volume, TE/TR $=5.2 / 15.4 \mathrm{~ms}$ and flip angle $=9^{\circ}$. For the third volume, $\mathrm{TE} / \mathrm{TR}=5.4 / 18.4 \mathrm{~ms}$ and flip angle $=9^{\circ}$. For the fourth volume, TE $/ \mathrm{TR}=5.0 / 14 \mathrm{~ms}$ and flip angle $=9^{\circ}$. 

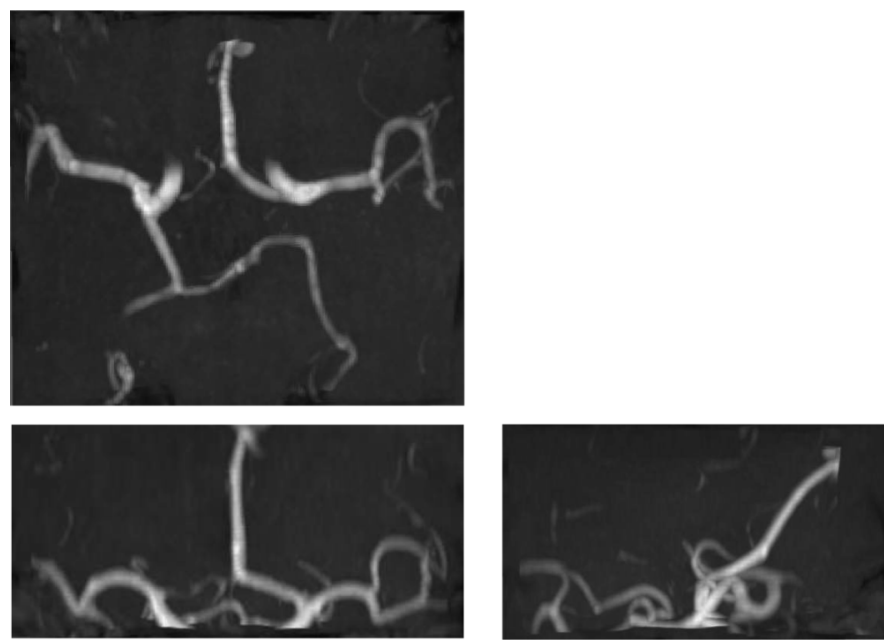

Fig. 9. The MIP images of a TOF-MRA image volume (First row: axial projection; second row: coronal projection and saggital projection). This TOF-MRA image volume has dimension $188 \times 168 \times 39$ voxels and voxel size $0.4 \mathrm{~mm} \times 0.4 \mathrm{~mm} \times 1.0 \mathrm{~mm}$.
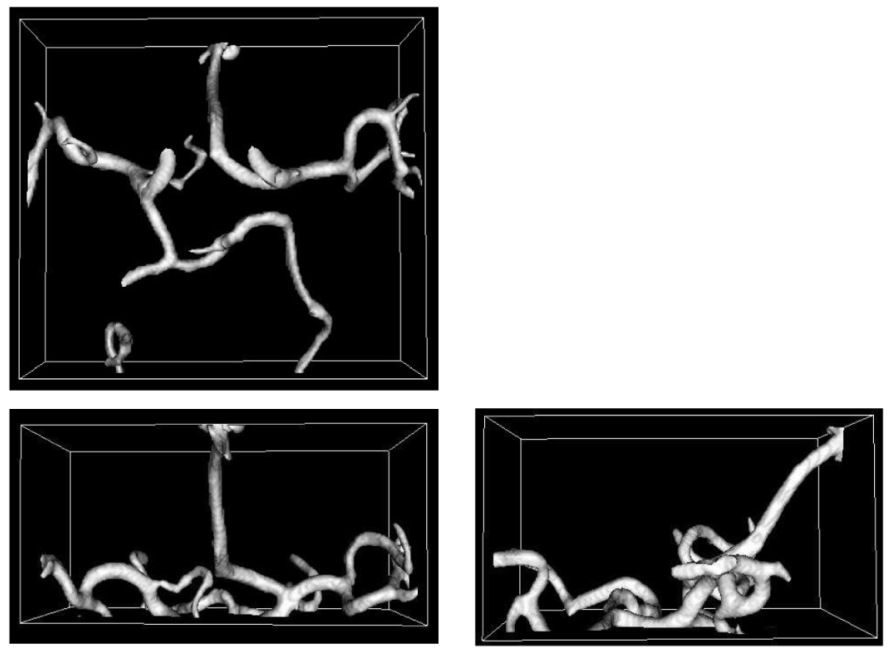

Fig. 10. Segmentation results of WLV-FLUX.

In these four experiments, the set of detection radii $R$ for both FLUX and WLV-FLUX [(16) and (19)] was set to 0.4, 0.8, 1.2, $1.6,2$, and $2.4 \mathrm{~mm}$. Due to the presence of narrow vessels, which involved a few voxels across the boundaries, the parameters of FLUX and WLV-FLUX were chosen for the detection of vessels having the width of one voxel. Such parameters included the scale of the Gaussian smoothing operation for FLUX in (17), and $\sigma$ for WLV-FLUX used in (1). These two parameters were set to $0.3 \mathrm{~mm}$ (slightly smaller than the $x-y$ plane voxel spacing). For $\sigma_{\perp}$ of WLV-FLUX, the value of $1.0 \mathrm{~mm}$ (the slice spacing) was chosen. It aims to guarantee that the filters used in (2) have an effective range to cover adequate number of voxels in all directions $\mathbf{n}$ for (4) and (5).

The smoothness regularization term $\beta$ in (20) was set to 0.05 for WLV-FLUX in order to maintain the contour smoothness. In FLUX, we observe that this regularization term can neutralize the effect of the evolution speed term in (20) and, hence, can halt contours at the undesired positions. Since this term is not necessary to be beneficial for the segmentation results of
FLUX, we present the best results obtained from FLUX using $\beta=0$ and $\beta=0.05$.

For these clinical cases, the same set of initial seed points for WLV-FLUX and FLUX was produced by global thresholding of each image volume. The thresholds were selected carefully to ensure that the seed points were located inside the major vessels to provide proper initial conditions to start the both algorithms, WLV-FLUX and FLUX. The algorithms were then stopped when the accumulated per-voxel update of the level set function was less than $10^{-4}$ after ten iterations.

In the first experiment on TOF-MRA image volume, as shown in Figs. 9 and 10, based on visual assessment between MIP images and segmented images, WLV-FLUX selects most of the vessels from the image volume and its results have no leakage. Conversely, the segmentation results of FLUX have leakages at several positions, as shown in Fig. 11(a). The results of FLUX with regularization parameter $\beta=0.05$ are presented because, when $\beta=0.05$, it encourages a smooth resultant contour and showed less leakages compared with the results using $\beta=$ 0 for this image volume. However, leakages still occurred at three positions in a low contrast branch. The leakage positions of FLUX are pointed at by three arrows in the axial view of Fig. 11(a) (top-left). In Fig. 11(b) and (c), four slices of these leakage regions are shown and the positions of leakages are indicated by arrows. At these positions, contours propagated through the weak vessel boundaries and this resulted in leakages. The leakages continued to expand and contaminated the results in other regions. This leads to difficulty in visualizing the results. Therefore, instead of presenting the final segmentation results of FLUX, the results shown in Fig. 11 are an intermediate step that leakages just began. In the axial projection of Fig. 9 (top-left), there is a vessel segment at the bottom-right position missed by FLUX, as shown in Fig. 11(a). It should be pointed out that FLUX can discover this segment after a number of iterations of contour evolution. However, such results including this segment was contaminated by the leaked contours and cannot be shown here.

The cause of the leakages is that the weak vessel boundaries cannot halt the FLUX contour, which can be attracted by the boundaries of other tissues attached on the vessels. In WLV-FLUX, the edge strength estimated from WLV-EDGE retains large values in weak boundaries so that the contour is able to stop on these weak boundaries and the vasculature is successfully captured without leakage.

In the second experiment, as shown in Fig. 12, a PC-MRA image volume was used. The results of FLUX using $\beta=0$ for (20) are presented. The use of $\beta=0$ disables the smoothness regularization term and encourages contour to evolve into branches. Even though this regularization term was disabled, FLUX missed some branches, as shown in Fig. 14(a). The missing branches are pointed at by arrows in Fig. 14(b) and (c). Fig. 13 shows that the segmentation results of WLV-FLUX are satisfactory, and the low intensity branches are included in the segmentation results.

For the third experiment, another PC-MRA image volume was used, as shown in Fig. 15. Similar to the previous experiment, the results of FLUX using $\beta=0$ for (20) are presented. It is observed that FLUX can only locate the major vessels, 


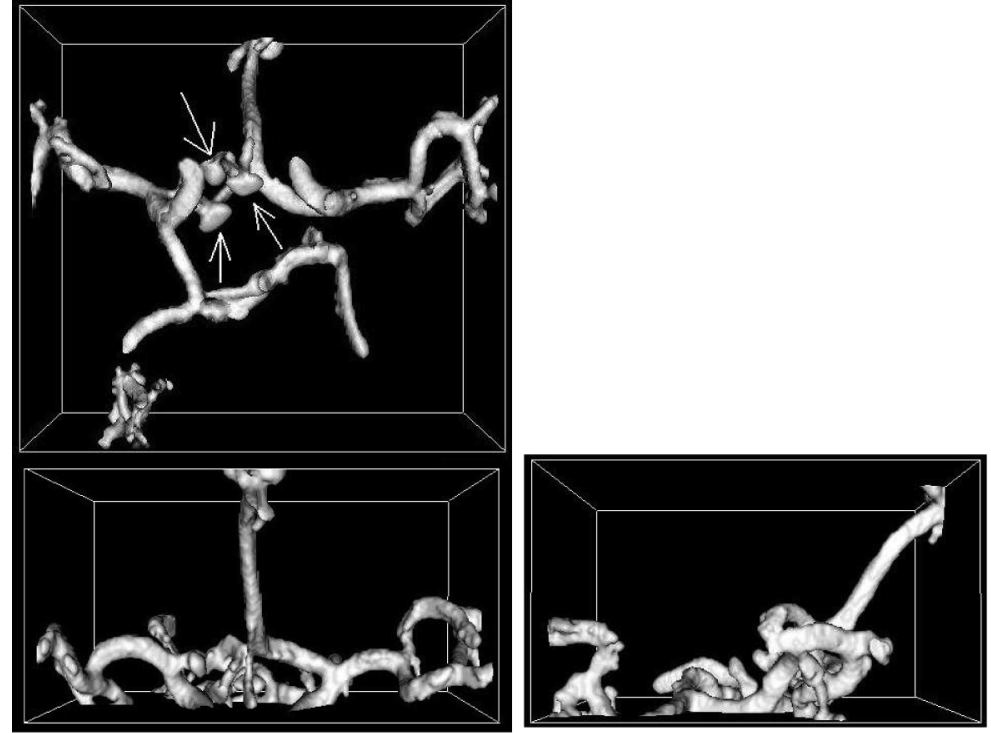

(a)
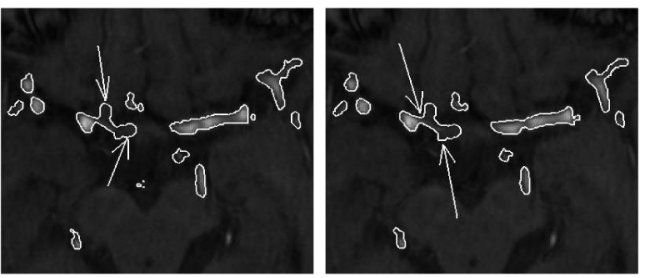

(b)
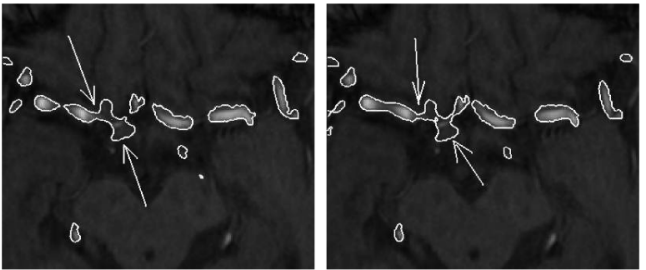

(c)

Fig. 11. (a) Segmentation results of FLUX. (b) The 14th and 15th axial slices. (c) The 11th and 12th axial slices of the TOF-MRA image volume and the contours

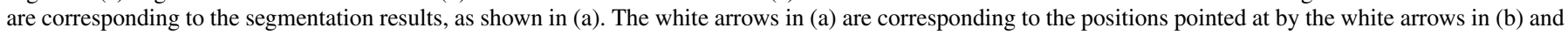
(c) where leakages occur.
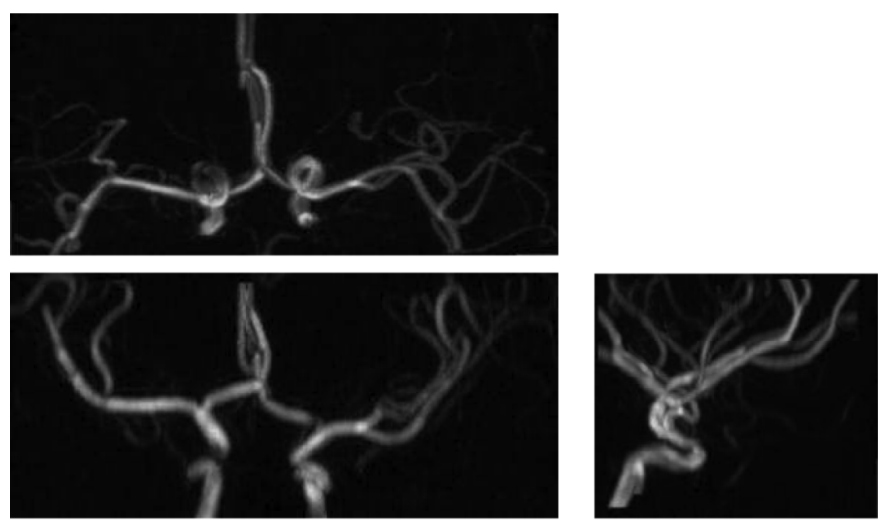

Fig. 12. The MIP images of a PC-MRA speed image volume (First row: axial projection; second row: coronal projection and saggital projection). This image volume has dimension $130 \times 286 \times 52$ voxels and voxel size $0.4 \mathrm{~mm} \times 0.4$ $\mathrm{mm} \times 1.0 \mathrm{~mm}$

as shown in Fig. 17(a). The missing branches are pointed at by arrows in Fig. 17(b) and (c). In contrast, better segmentation results are obtained using WLV-FLUX where low contrast branches are captured, as shown in Fig. 16.

In Fig. 14(b) and (c) and Fig. 17(b) and (c), the image slices show the white arrows pointing at the low contrast vessels missed by FLUX in the second and third experiments. As shown in these figures, the contours are halted inside the branches rather than the boundaries of the branches. The main reason is that the boundaries of the low contrast branches do not provide gradient magnitude strong enough to attract contours for propagation. For WLV-FLUX, the contours can evolve into the low contrast vessels because WLV-EDGE is capable of returning large and consistent edge strength on those low contrast boundaries.

Finally, a PC-MRA image volume, as shown in Fig. 18, was utilized in the forth segmentation experiment. The results of
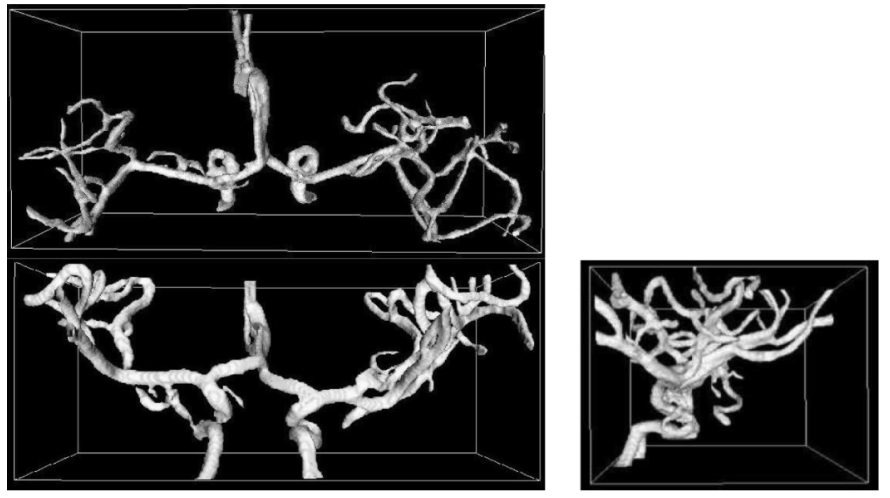

Fig. 13. Segmentation results of WLV-FLUX.

FLUX using $\beta=0.05$ for (20) are presented. When $\beta=0.05$, it reduces the chance of leakages by encouraging smooth resultant contours. In this case, both FLUX and WLV-FLUX are able to capture the vessels, as shown in Figs. 19 and 20. Nonetheless, WLV-FLUX gives better segmentation results in the low contrast branches compared with FLUX because, for FLUX, leakages occurred in the low contrast branches indicated by the arrows in Figs. 20 and 21. The low contrast vessel boundaries could not halt the contours accurately, which were attracted by the noise near the vessel boundaries. Unlike the first experiment, which shows an intermediate evolution step for FLUX (see Fig. 11), the results shown in Fig. 20 are the final segmentation results obtained using FLUX as the leakages in this experiment were stopped before they contaminated the results in other regions. Compared with FLUX, WLV-FLUX is able to halt the contours at the low contrast vessel boundaries.

We have measured the computation times required for segmenting blood vessels using WLV-FLUX and FLUX in the 

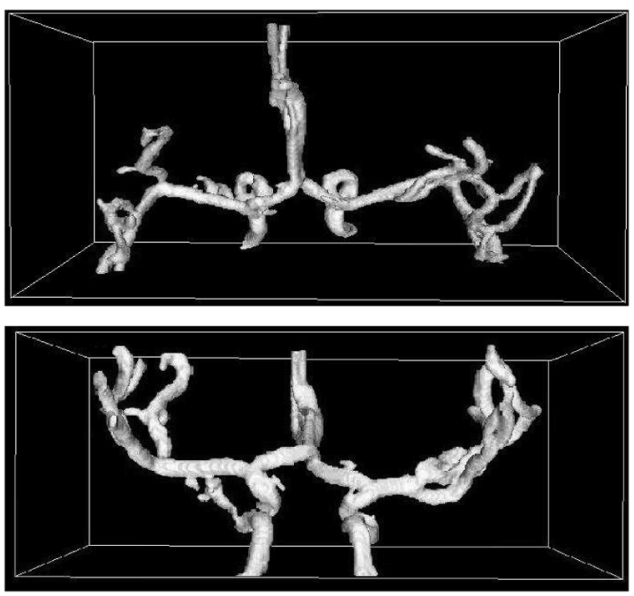

(a)
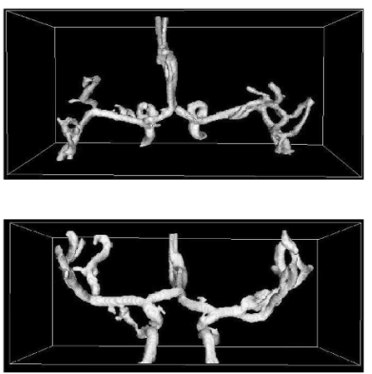

(b)
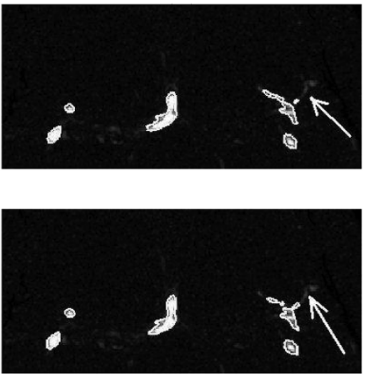

(c)

Fig. 14. Segmentation results of FLUX. (b) The 26th and 27th axial slices. (c) The 40th and 41st axial slices of the PC-MRA image volume and the contours are corresponding to the segmentation results, as shown in (a). The white arrows in (b) and (c) indicate the low contrast vessels, which are missed by FLUX.
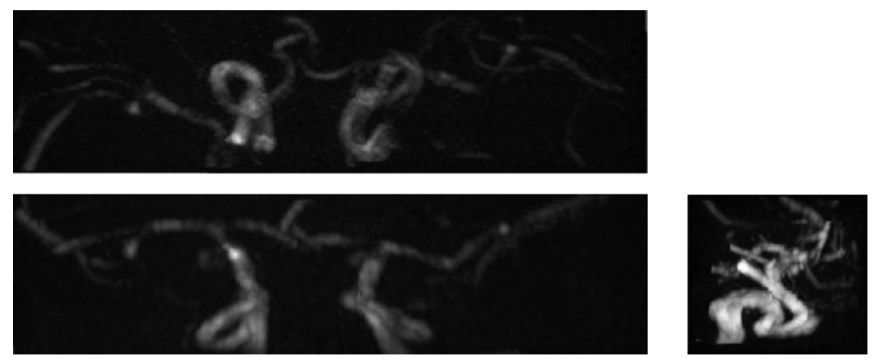

Fig. 15. The MIP images of a PC-MRA speed image volume (First row: axial projection; second row: coronal projection and saggital projection). This image volume has dimension $67 \times 257 \times 35$ voxels and voxel size $0.4 \mathrm{~mm} \times 0.4$ $\mathrm{mm} \times 0.8 \mathrm{~mm}$.

above four clinical image volumes. For WLV-FLUX, the measured time includes the computation of WLV-EDGE and estimation of $F$ in (20). For FLUX, the measured time includes the computation of GRADIENT and estimation of $F$ in (20). The implementations of both WLV-FLUX and FLUX were based on programs written using Microsoft Visual C++ in Windows XP 32-bit environment and run on a Pentium IV 3.2 GHz PC with 1 GB RAM. As the image volumes have different sizes, rather than the average running time, the individual running times are reported in this paper. For WLV-FLUX, the running times for the first, second, third, and forth experiments were $3711,6449,1924$, and 5634 s, respectively. For FLUX, the running times were $3500,5737,1725$, and $5441 \mathrm{~s}$, respectively. It is observed that the running time for WLV-FLUX is only slightly longer (around 9\%) than FLUX.
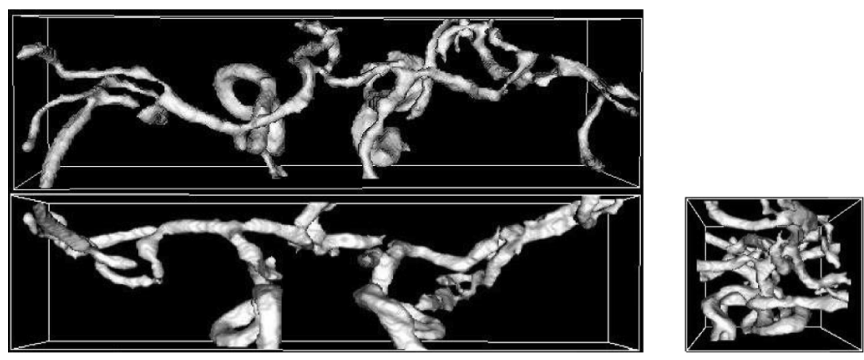

Fig. 16. Segmentation results of WLV-FLUX.

\section{DisCUSSION}

\section{A. Edge Detectors}

The main contribution of this paper is proposing the use of WLV to quantify the intensity similarity between two sides of an edge. For boundary detection, this intensity similarity measure is complemented with the intensity difference across an edge to formulate a new WLV-based edge detection method, namely, WLV-EDGE. It is shown to be robust to the change of intensity contrast of edges and that it can give high and consistent edge strength for low contrast boundaries. As such, this method is ideal for handling low contrast vessel boundaries. Since the proposed method considers intensity similarity of local regions between both sides of an edge, it is different from other differential operators for edge detection, such as Sobel filters, Roberts filters, Prewitt filters, filters used by Canny edge detection scheme [5], $\lambda_{\tau}$-Space Representation [10], quadrature filters [13], difference of a Gaussian [16], Laplacian of Gaussian [18], as well as the Hessian matrix. Their detection principles rely entirely on the intensity change 


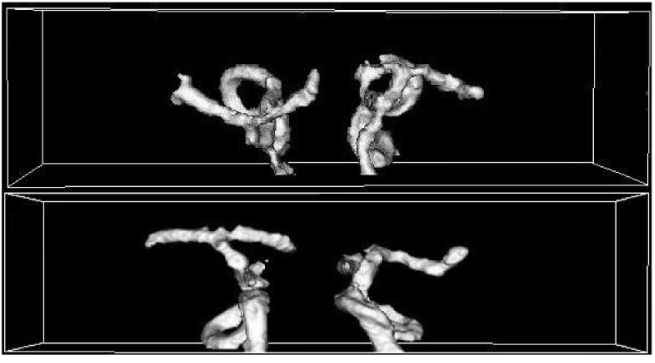

(a)
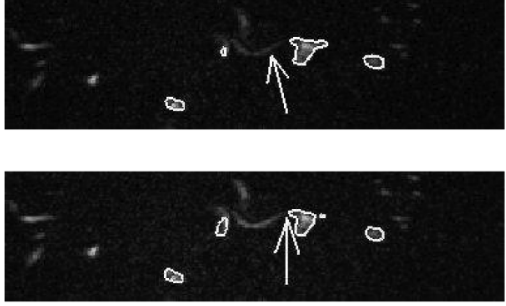

(b)
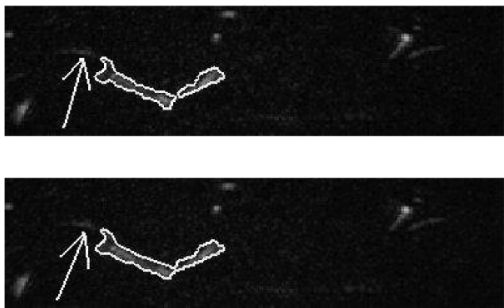

(c)

Fig. 17. Segmentation results of FLUX. (b) The 25th and 26th axial slices. (c) The 29th and 30th axial slices of the PC-MRA image volume and the contours are corresponding to the segmentation results, as shown in (a). The white arrows in (b) and (c) indicate the low contrast vessels, which are missed by FLUX.
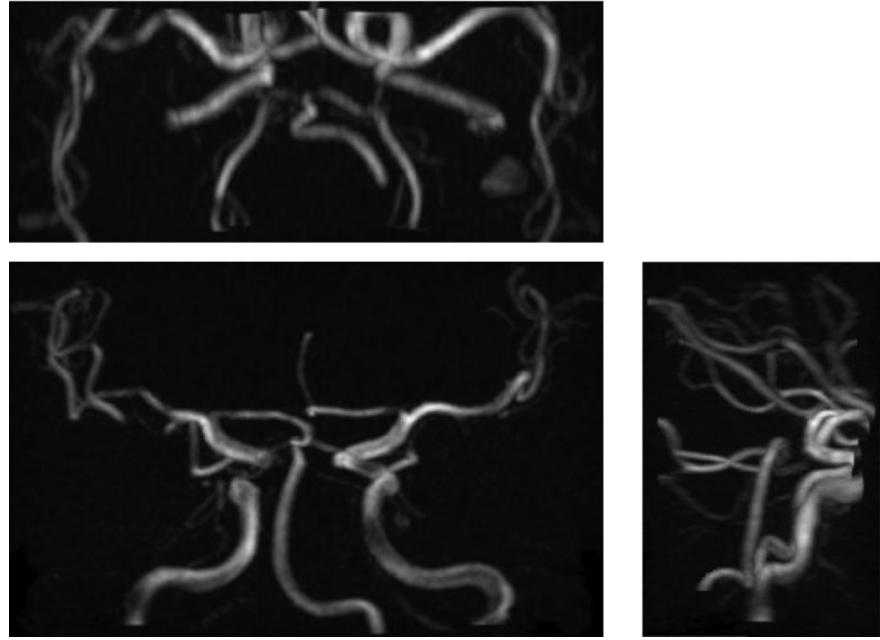

Fig. 18. The MIP images of a PC-MRA speed image volume (First row: axial projection; second row: coronal projection and saggital projection). This image volume has dimension $104 \times 252 \times 64$ voxels and voxel size $0.4 \mathrm{~mm} \times 0.4 \mathrm{~mm} \times 1.0 \mathrm{~mm}$

across boundary, and therefore, they can only return small edge strength responses at low contrast edges. Our method, on the other hand, not only relies on the intensity change across boundary but also takes into account the intensity homogeneity in two local regions separated by an edge.

\section{B. Edge Detection and Segmentation}

In our experiments, the segmentation results of WLV-FLUX are compared against FLUX. The major distinction between FLUX and WLV-FLUX is that they use different edge information. FLUX utilizes the smoothed image gradient (17) and WLV-FLUX employs WLV-EDGE (18). The mathematical comparison between the smoothed image gradient and the confidence value [as defined in (5)] of WLV-EDGE is provided in
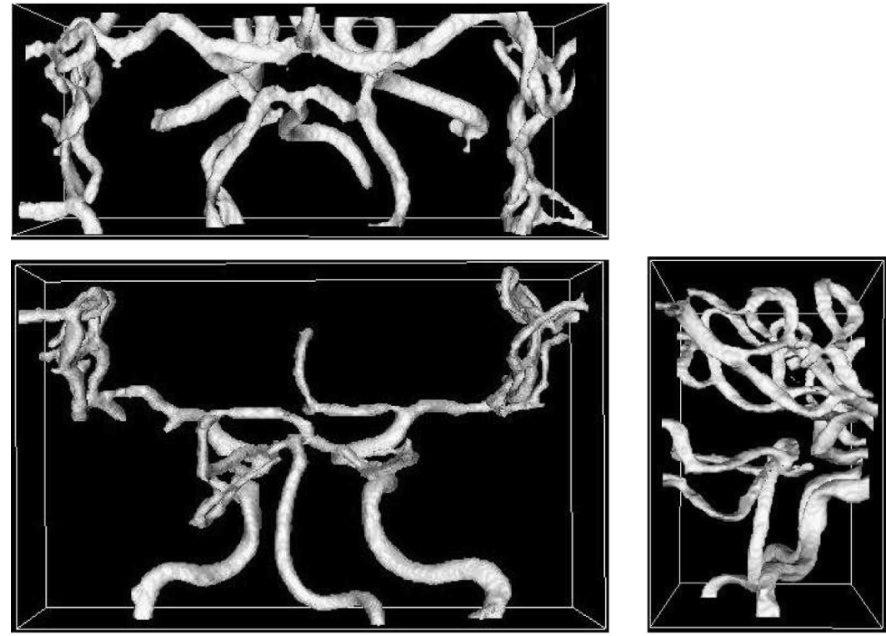

Fig. 19. Segmentation results of WLV-FLUX
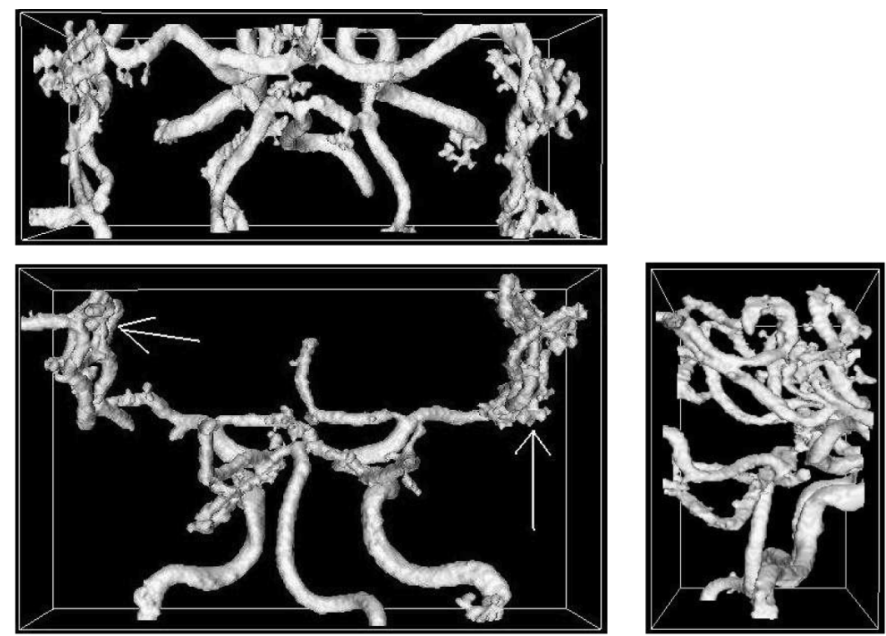

Fig. 20. Segmentation results of FLUX 

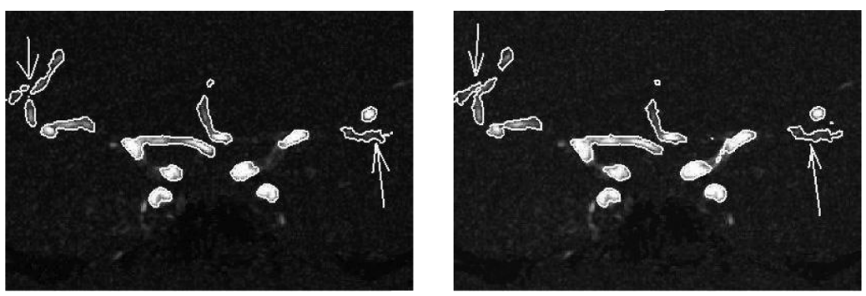

(a)
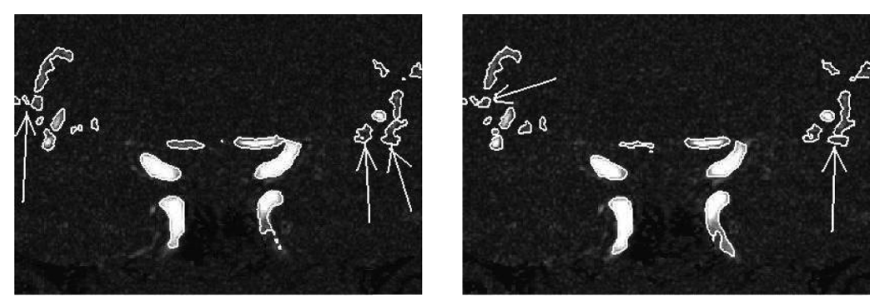

(b)

Fig. 21. Segmentation results of FLUX. (a) The 17th and 18th coronal slices. (b) The 25th and 26th coronal slices of the PC-MRA image volume and the contours are corresponding to the segmentation results, as shown in Fig. 20. The white arrows in (a) and (b) indicate the low contrast vessels, which are missed by FLUX.

Appendix-A, where the confidence value is written as

$$
R_{\hat{n}}(\vec{x})=\left(\frac{1}{Z}\right)\left(\frac{f_{\hat{n}}(\vec{x}) * I(\vec{x})}{\sqrt{\min \left(\mathrm{WLV}_{1, \hat{n}}(\vec{x}), \mathrm{WLV}_{2, \hat{n}}(\vec{x})\right)}+\epsilon}\right)
$$

where $Z=-\int f_{1, \hat{n}}(\vec{u}) d \vec{u}=\int f_{2, \hat{n}}(\vec{u}) d \vec{u}$ is a constant. In the above equation, the confidence value $R_{\hat{n}}(\vec{x})$ of WLV-EDGE is composed of three parts: a constant $Z^{-1}$; the numerator, which is based on the anisotropically $\left(\sigma \neq \sigma_{\perp}\right)$ or isotropically $(\sigma=$ $\left.\sigma_{\perp}\right)$ smoothed image gradient along direction $\hat{n}$; and the denominator, which involves WLVs. Analogous to the discussion in Section II-B, the denominator, which is governed by the values of WLV, enables the confidence value of WLV-EDGE to: (a) be more robust against the change of intensity contrast and (b) have strong and consistent edge strength responses on low contrast edges. Therefore, WLV-EDGE enables WLV-FLUX to capture the low contrast branches in the experiments but such branches are not discovered by FLUX.

It is worth mentioning that smoothing image prior to the calculation of image gradient as suggested in the gradient-based approaches [6], [12], [14], [15], [17], [19], [20], [24], [25], [26], [27], [29], [30], [31], [32], [33] i.e., $\nabla(G * I)=(\nabla G) * I$, yields a connection to the filters used in Canny edge detector [5]. This is because they are grounded on applying directional derivatives of a Gaussian function along the $x, y$, and $z$ directions in an image. As such, the calculation of image gradient can be viewed as a procedure of edge detection prior to performing segmentation. To segment vessels in low contrast regions with the presence of intensity inhomogeneity, these segmentation methods need an edge detector, which is able to detect low contrast edges. Therefore, WLV-EDGE, which returns high and consistent edge strength for low contrast edges, is suitable for segmentation of vessels in low contrast regions.

\section{Future Extension-Shape and Structural Information}

In the current stage, the proposed method does not analyze the shape and local structures of boundaries. Currently, the focus of this paper is to propose and validate a framework for boundary detection based on: (a) intensity similarity among regions on both sides of an edge and (b) intensity difference across the edge, without considering the shape and local structures of boundaries. Utilizing shape information or structural information along with WLV-EDGE to provide more advanced features for vessel boundary detection is the future direction of this research. For instance, the eigenvalues along different principle directions of the matrix stated in (10) can provide structural information of boundaries; the proposed method can be combined with the Hessian matrix (details are provided in Appendix B) for the analysis of the shape and local structures of boundaries; using the minimum curvature instead of the mean curvature in (20), i.e., using the codimension two level set approach [1], can enhance the accuracy of WLV-FLUX in the extraction of vessels in tubular shape.

\section{CONCLUSION}

In summary, the robustness of WLV-EDGE against the change of intensity contrast of edges, and the capability of returning high and consistent edge strength on low contrast edges are shown to be beneficial for the segmentation of vasculatures in low contrast regions. Such robustness of WLV-EDGE is validated in experiments using three synthetic volumes. Moreover, in the presence of intensity inhomogeneity, such as bias field, the presence of other tissues or reduced speed related vessel intensity for narrow vessels in PC-MRA, WLV-FLUX makes use of the edge information of WLV-EDGE and is less likely than FLUX to have leakages or discontinuities in the four clinical segmentation experiments. It is experimentally shown that the WLV-based edge detection approach can achieve high quality segmentation of vasculatures in MRA.

\section{APPENDIX}

\section{A. Relationship Between Confidence Value and the Smoothed Image Gradient}

The term of intensity average difference in (5) can be written as

$$
\begin{aligned}
\mu_{1}-\mu_{2} & =\int\left(g_{1, \hat{n}}(\vec{y})-g_{2, \hat{n}}(\vec{y})\right) I(\vec{x}+\vec{y}) d \vec{y}, \\
& =\int\left(\frac{f_{1, \hat{n}}(\vec{y})}{\int f_{1, \hat{n}}(\vec{u}) d \vec{u}}-\frac{f_{2, \hat{n}}(\vec{y})}{\int f_{2, \hat{n}}(\vec{u}) d \vec{u}}\right) I(\vec{x}+\vec{y}) d \vec{y}
\end{aligned}
$$

since the directional derivative of a Gaussian function is antisymmetric, we define $Z$, i.e., $Z=-\int f_{1, \hat{n}}(\vec{u}) d \vec{u}=$ $\int f_{2, \hat{n}}(\vec{u}) d \vec{u}$ [see (1)-(3)]

$$
\begin{aligned}
& =-\frac{1}{Z} \int\left(f_{1, \hat{n}}(\vec{y})+f_{2, \hat{n}}(\vec{y})\right) I(\vec{x}+\vec{y}) d \vec{y} \\
& =-\frac{1}{Z} \int f_{\hat{n}}(\vec{y}) I(\vec{x}+\vec{y}) d \vec{y} \\
& =\frac{1}{Z} f_{\hat{n}}(\vec{x}) * I(\vec{x})
\end{aligned}
$$




$$
\mathbf{H}_{\mathrm{WLV}-\mathrm{EDGE}}=\left(\begin{array}{lll}
\left(\vec{w} \cdot(1,0,0)^{T}\right)_{x} & \left(\vec{w} \cdot(1,0,0)^{T}\right)_{y} & \left(\vec{w} \cdot(1,0,0)^{T}\right)_{z} \\
\left(\vec{w} \cdot(0,1,0)^{T}\right)_{x} & \left(\vec{w} \cdot(0,1,0)^{T}\right)_{y} & \left(\vec{w} \cdot(0,1,0)^{T}\right)_{z} \\
\left(\vec{w} \cdot(0,0,1)^{T}\right)_{x} & \left(\vec{w} \cdot(0,0,1)^{T}\right)_{y} & \left(\vec{w} \cdot(0,0,1)^{T}\right)_{z}
\end{array}\right) .
$$

where $*$ represents the convolution operator. Therefore, the intensity average difference of the numerator in (5) is equal to the results of convolving the directional derivative of a Gaussian function $f_{\hat{n}}(\vec{x})$ with a multiplicative constant $Z^{-1}$

$$
\begin{aligned}
R_{\hat{n}}(\vec{x}) & =\frac{\mu_{1, \hat{n}}(\vec{x})-\mu_{2, \hat{n}}(\vec{x})}{\sqrt{\min \left(\mathrm{WLV}_{1, \hat{n}}(\vec{x}), \mathrm{WLV}_{2, \hat{n}}(\vec{x})\right)}+\epsilon} \\
& =\left(\frac{1}{Z}\right) \frac{f_{\hat{n}}(\vec{x}) * I(\vec{x})}{\sqrt{\min \left(\mathrm{WLV}_{1, \hat{n}}(\vec{x}), \mathrm{WLV}_{2, \hat{n}}(\vec{x})\right)}+\epsilon}
\end{aligned}
$$

thus, the confidence value, as defined in (5), can be written in terms of the smoothed image gradient $\nabla(G(\vec{x}) * I(\vec{x}))$ if $\sigma=\sigma_{\perp}$

$$
\begin{aligned}
& =\left(\frac{1}{Z}\right)\left(\frac{\frac{\partial}{\partial \hat{n}}(G(\vec{x}) * I(\vec{x}))}{\sqrt{\min \left(\mathrm{WLV}_{1, \hat{n}}(\vec{x}), \mathrm{WLV}_{2, \hat{n}}(\vec{x})\right)}+\epsilon}\right) \\
& =\left(\frac{1}{Z}\right)\left(\frac{\hat{n} \cdot \nabla(G(\vec{x}) * I(\vec{x}))}{\sqrt{\min \left(\mathrm{WLV}_{1, \hat{n}}(\vec{x}), \mathrm{WLV}_{2, \hat{n}}(\vec{x})\right)}+\epsilon}\right) .
\end{aligned}
$$

\section{B. Incorporating WLV-EDGE in the Hessian Matrix}

It is straightforward to utilize the Hessian matrix to complement with WLV-EDGE. It can be achieved by rewriting the Hessian matrix $\mathbf{H}$ as follows:

$$
\begin{aligned}
\mathbf{H} & =\left(\begin{array}{lll}
(G * I)_{x x} & (G * I)_{x y} & (G * I)_{x z} \\
(G * I)_{y x} & (G * I)_{y y} & (G * I)_{y z} \\
(G * I)_{z x} & (G * I)_{z y} & (G * I)_{z z}
\end{array}\right) \\
& =\left(\begin{array}{lll}
\left(G_{x} * I\right)_{x} & \left(G_{x} * I\right)_{y} & \left(G_{x} * I\right)_{z} \\
\left(G_{y} * I\right)_{x} & \left(G_{y} * I\right)_{y} & \left(G_{y} * I\right)_{z} \\
\left(G_{z} * I\right)_{x} & \left(G_{z} * I\right)_{y} & \left(G_{z} * I\right)_{z}
\end{array}\right)
\end{aligned}
$$

and then replacing the first derivative operation of the Hessian matrix with the edge detection results of WLV-EDGE in (18), i.e., see (28) shown at top of the page. As such, the robustness of WLV-EDGE against changes of intensity contrast can be inherited in the Hessian matrix.

\section{ACKNOWLEDGMENT}

The authors wish to thank Dr. P. Summers for providing clinical data and would like to thank anonymous reviewers for the constructive comments.

\section{REFERENCES}

[1] L. Ambrosio and H. M. Soner, "Level set approach to mean curvature flow in arbitrary codimension," J. Differential Geometry, vol. 41, no. 4, pp. 693-737, 1996.

[2] B. AubertBroche, A. C. Evans, and L. Collins, "A new improved version of the realistic digital brain phantom," Neurolmage, vol. 32, no. 1, pp. 138-145, 2006.
[3] J. Bigiin, "Multidimensional orientation estimation with applications to texture analysis and optical flow," Trans. Pattern Anal. Machine Intell., vol. 13, no. 8, pp. 775-790, Aug. 1991.

[4] E. Bullitt, S. Aylward, A. Liu, J. Stone, S. Mukherji, C. Coffey, G. Gerig, and S. M. Pizer, "3D graph description of the intracerebral vasculature from segmented MRA and tests of accuracy by comparison with x-ray angiograms," in Proc. Int. Conf. Inf. Process. Med. Imag., 1999, vol. 1613, pp. 308-321.

[5] J. Canny, "A computational approach to edge detection," IEEE Trans. Pattern Anal. Machine Intell., vol. 8, no. 61, pp. 679-697, 1986.

[6] V. Caselles, R. Kimmel, and G. Sapiro, "Geodesic active contours," Int. J. Comput. Vision, vol. 22, no. 1, pp. 61-79, 1997.

[7] C. A. Cocosco, V. Kollokian, R. K.-S. Kwan, and A. C. Evans, "BrainWeb: Online interface to a 3D MRI simulated brain database," in Proc. Int. Conf. Functional Mapping of the Human Brain, 1997, vol. 5, p. 425. [Online]. Available: http://www.bic.mni.mcgill.ca/brainweb/

[8] M. Descoteaux, M. Audette, K. Chinzei, and K. Siddiqi, "Bone enhancement filtering: Application to sinus bone segmentation arid simulation of pituitary surgery," in Proc. Int. Conf. Med. Image Comput. Comput. Assisted Intervention, 2005, pp. 9-16.

[9] A. Frangi, W. Niessen, and M. A. Viergever, "Multiscale vessel enhancement filtering," in Proc. Int. Conf. Med. Image Comput. Comput. Assisted Intervention, 1998, pp. 130-137.

[10] M. Gokmen and A. K. Jain, "AT-space representation of images and generalized edge detector," IEEE Trans. Pattern Anal. Machine Intell., vol. 19, no. 6, pp. 545-563, 1997.

[11] L. Ibanez, W. Schroeder, L. Ng, and J. Gates, The ITK Software Tool Kit.

[12] S. Kichenassamy, A. Kimar, P. J. Olver, A. Tannenbaum, and A. J. Yezzi, "Gradient flows and geometric active contour models," in Proc. Int. Conf. Comput. Vision, 1995, pp. 810-815.

[13] H. Knutsson, "Representing local structure using tensors," in Proc. Scandinavian Conf. Image Anal., 1989, pp. 244-251.

[14] L. M. Lorigo, O. Faugeras, W. E. L. Crimson, R. Keriven, R. Kikinis, A. Nabavi, and C.-F. Westin, "Codimension-Two geodesic active contours for the segmentation of tubular structures," in Proc. IEEE Conf. Comput. Vision Pattern Recogn., 2000, vol. 1, pp. 444-451.

[15] L. M. Lorigo, O. Faugeras, W. E. L. Crimson, R. Keriven, R. Kikinis, A. Nabavi, and C.-F. Westin, "Curves: Curve evolution for vessel segmentation," Med. Image Anal., vol. 5, no. 3, pp. 195-206, 2001.

[16] W. Ma and B. Manjunath, "Edgeflow: A technique for boundary detection and image segmentation," IEEE Trans. Image Process., vol. 9, no. 8 , pp. $1375-1388$, Aug. 2000.

[17] R. Malladi, J. Sethian, and B. Vemuri, "Shape modeling with front propagation: A level set approach," IEEE Trans. Pattern Anal. Machine Intell., vol. 17, no. 2, pp. 158-175, Feb. 1995.

[18] D. Marr and E. Hildreth, "Theory of edge detection," in Proc. Royal Soc. London (B), 1980, vol. 207, pp. 187-217.

[19] T. Mclnerney and D. Terzopoulos, "Topology adaptive deformable surfaces for medical image volume segmentation," IEEE Trans. Med. Imag., vol. 18 , no. 10 , pp. 840-850, 1999.

[20] H. T. Nguyen, M. Worring, and R. van den Boomgaard, "Watersnakes: Energy-driven watershed segmentation," IEEE Trans. Pattern Anal. Machine Intell., vol. 25, no. 3, pp. 330-342, Mar. 2003.

[21] S. Osher and J. Sethian, "Fronts propagating with curvature dependent speed: Algorithms based on Hamilton-Jacobi formulations," $J$. Comput. Phys., vol. 79, no. 1, pp. 12-49, 1988.

[22] Y. Sato, S. Nakajima, N. Shiraga, H. Atsumil, S. Yoshida, T. Koller, G. Gerig, and R. Kikinis, "Three-dimensional multi-scale line filter for segmentation and visualization of curvilinear structures in medical images," Med. Image Anal., vol. 2, no. 2, pp. 143-168, 1998.

[23] M. Styner, C. Brechbuhler, G. Szekely, and G. Gerig, "Parametric estimate of intensity inhomogeneities applied to MRI," IEEE Trans. Med. Imag., vol. 19, no. 3, pp. 153-165, Mar. 2000.

[24] J. S. Suri, K. Liu, S. Singh, S. N. Laxminarayan, X. Zeng, and L. Reden, "Shape recovery algorithms using level sets in 2-D/3-D medical imagery: A state-of-the-art review," IEEE Trans. Inf. Technol. Biomed., vol. 6 , no. 1 , pp. 8-28, Mar. 2002. 
[25] A. Vasilevskiy and K. Siddiqi, "Flux maximizing geometric flows," IEEE Trans. Pattern Anal. Machine Intell., vol. 24, no. 12, pp. 1565-1578, Dec. 2002.

[26] K. C. Wang, R. W. Dutton, and C. A. Taylor, "Three-dimensional multi-scale line filter for segmentation and visualization of curvilinear structure in medical images," IEEE Eng. Med. Biol. Mag., vol. 18, no. 6, pp. 33-39, 1999.

[27] C. F. Weslin, L. M. Lorigo, O. Faugeras, W. E. L. Crimson, S. Dawson, A. Norbash, and R. Kikinis, "Segmentation by adaptive geodesic active contours," in Proc. Int. Conf. Med. Image Comput. Comput. Assisted Intervention, 2000, pp. 266-275.

[28] R. Whitaker, "A level-set approach to 3D reconstruction from range data," Int. J. Comput. Vision, vol. 29, no. 33, pp. 203-231, 1998.

[29] Y. Xiang, A. C. S. Chung, and J. Ye, "A new active contour method based on elastic interaction," in Proc. IEEE Conf. Comput. Vision d Pattern Recogn., 2005, vol. 1, pp. 452-457.
[30] Y. Xiang, A. C. S. Chung, and J. Ye, "An active contour model for image segmentation based on elastic interaction," J. Comput. Phys., vol. 219, no. 1, pp. 455-476, 2006.

[31] P. Yan and A. A. Kassim, "MRA image segmentation with capillary active contour," in Proc. Int. Conf. Med. Image Comput. Comput. Assisted Intervention, 2005, pp. 51-58.

[32] P. Yan and A. A. Kassim, "Segmentation of volumetric MRA images by using capillary active contour," Med. Image Anal., vol. 10, no. 3, pp. 317-329, 2006

[33] A. J. Yezzi, S. Kichenassamy, A. Kumar, P. Olver, and A. Tannenbaum, "A geometric snake model for segmentation of medical imagery," IEEE Trans. Med. Imag., vol. 16, no. 2, pp. 199-209, Apr. 1997. 